\title{
Lusioersily
}

\section{Preparation, characterization and in-vitro efficacy of quercetin loaded liquid crystalline nanoparticles for the treatment of asthma}

Cherk Yong, D. O., Saker, S. R., Wadhwa, R., Chellappan, D. K., Madheswaran, T., Panneerselvam, J.,

Tambuwala, M. M., Bakshi, H. A., Kumar, P., Pillay, V., Gupta, G., Oliver, B. G., Wark, P., Hsu, A., Hansbro, P. M., Dua, K., \& Zeeshan, F. (2019). Preparation, characterization and in-vitro efficacy of quercetin loaded liquid crystalline nanoparticles for the treatment of asthma. Journal of Drug Delivery Science and Technology, 54, [101297]. https://doi.org/10.1016/j.jddst.2019.101297

Link to publication record in Ulster University Research Portal

Published in:

Journal of Drug Delivery Science and Technology

Publication Status:

Published (in print/issue): 31/12/2019

DOI:

10.1016/j.jddst.2019.101297

Document Version

Author Accepted version

\section{General rights}

Copyright for the publications made accessible via Ulster University's Research Portal is retained by the author(s) and / or other copyright owners and it is a condition of accessing these publications that users recognise and abide by the legal requirements associated with these rights.

\section{Take down policy}

The Research Portal is Ulster University's institutional repository that provides access to Ulster's research outputs. Every effort has been made to ensure that content in the Research Portal does not infringe any person's rights, or applicable UK laws. If you discover content in the Research Portal that you believe breaches copyright or violates any law, please contact pure-support@ulster.ac.uk. 


\title{
Manuscript Details
}

\section{Manuscript number}

Title
JDDST_2019_1287_R1

Preparation, characterization and in-vitro efficacy of quercetin loaded liquid crystalline nanoparticles for the treatment of asthma

Quercetin loaded liquid crystalline nanoparticles for the treatment of asthma

\section{Article type}

Research Paper

\begin{abstract}
The present study aims to formulate quercetin loaded liquid crystalline nanoparticles (LCN) and surface modified liquid crystalline nanoparticles (sm-LCN) as well as investigate their anti-inflammatory activity in human primary bronchial epithelial cell line (BCi-NS1.1) induced with lipopolysaccharide (LPS). Quercetin LCN were prepared using ultrasonication method. The formulated LCNs and sm-LCNs were characterised in terms of particle size, zeta potential as well as the drug encapsulation efficiency. Furthermore, their morphology and in vitro release profile were also studied. In addition, the anti-inflammatory activity of quercetin LCN and sm-LCNs were evaluated by measuring the concentration of pro-inflammatory markers namely interleukin (IL)-1 $\beta$, IL-6 and IL-8 in BCI-NS1.1 cell lines via cytometric bead array. The molecular mechanism inherent to the inclusion of quercetin into monoolein nanosystem and surface modification of the nanosystem with chitosan was elucidated via molecular mechanics simulations. Quercetin LCN and sm-LCN significantly $(p<0.05)$ decreased the production of IL-1 $\beta$, IL-6 and IL- 8 compared to LPS only group. Encapsulation of quercetin into LCN and sm-LCN further enhanced its anti-inflammatory activity compared to quercetin in dimethyl sulfoxide (DMSO). In addition to that, quercetin LCN and sm-LCN also exhibited comparable activity to fluticasone in terms of significantly $(p<0.05)$ reducing the production of IL-1 $\beta$ and IL-6. Quercetin loaded LCN and sm-LCN could be a potential therapeutic intervention for asthma as they are efficacious in suppressing the production of key pro-inflammatory cytokines associated with the development of asthma.
\end{abstract}

\section{Keywords}

Corresponding Author

Corresponding Author's Institution

Order of Authors

\section{Suggested reviewers}

Quercetin; Liquid crystalline nanoparticles; Asthma; Inflammation

Kamal Dua

University of Sydney Technology

David Ong Cherk Yong, Sanggetha Raja Saker, Ridhima Wadhwa, Dinesh Kumar Chellappan, Thiagarajan Madheswaran, Jithendra Panneerselvam, Murtaza Tambuwala, Hamid Bakshi, Pradeep Kumar, Viness Pillay, gaurav gupta, oliver brian, Peter Wark, Alan Hsu, Philip Hansbro, Kamal Dua, Farrukh Zeeshan

Pawan Maurya, Srinivas Nammi, Madhur Shastri, Shakti Shukla

\section{Submission Files Included in this PDF}

File Name [File Type]

Cover Letter.docx [Cover Letter]

Reviewer Comments.docx [Response to Reviewers]

Quercetin Revised.docx [Revised Manuscript with Changes Marked]

Graphical Abstract.jpg [Graphical Abstract]

Quercetin 6-9-2019.docx [Manuscript File]

Copy-of-Figures.docx [Figure]

Figures.docx [Figure]

Tables.docx [Table]

Conflict of interests.docx [Conflict of Interest] 
To view all the submission files, including those not included in the PDF, click on the manuscript title on your EVISE Homepage, then click 'Download zip file'.

\section{Research Data Related to this Submission}

There are no linked research data sets for this submission. The following reason is given:

No data was used for the research described in the article 
The Editor

Journal of Drug Delivery Science \& Technology

Subject: Regarding submission of an original research article for publication in Journal of Drug delivery Science and Technology entitled: Preparation, characterization and in-vitro efficacy of quercetin loaded liquid crystalline nanoparticles for the treatment of asthma.

\section{Dear Editor,}

Please find attached our original manuscript entitled "Preparation, characterization and in-vitro efficacy of quercetin loaded liquid crystalline nanoparticles for the treatment of asthma" for your kind consideration for publication in the Journal of Drug Delivery Science and Technology. The present research work is original and has not been published elsewhere, nor is it currently under consideration for publication elsewhere.

The manuscript discusses about bronchial asthma, being a chronic inflammatory disease of airways, has numerous treatment options, none of which has disease-modifying properties. Quercetin, a dietary flavonoid commonly found in fruits and vegetables exhibits a wide spectrum of pharmacological activities; prominent among which is its anti-inflammatory activity, which has been proved, as a potential therapeutic intervention in the treatment of bronchial asthma, by various in vitro and in vivo animal studies.

Targeted and controlled delivery of quercetin directly to the lungs is extremely difficult. The use of liquid crystalline nanoparticle as an intervention in asthma is still not fully explored and only minimal attempts have been made. This directs an utmost need and attention, to identify a potential drug delivery platform that have minimum side effects and maximum patient compliance. Keeping all these facts, we have designed and conducted our present unique and a significant study, where, we have developed quercetin-loaded liquid crystalline nanoparticles as a means of delivery. In addition, quercetin is well known for its poor bioavailability and warrants the use of nanoparticles to overcome those limitations along with providing a prolonged release of quercetin.

The prepared liquid crystalline nanoparticles were characterized for their physicochemical properties, invitro release study and in-vitro anti-inflammatory studies focussed on various pro-inflammatory markers using immortalized human airway basal cell line (BCi-NS1.1). The prepared liquid crystalline nanoparticles were found to be stable and exhibited a controlled release pattern along with the suppression of various antiinflammatory genes clearly demonstrating the potential of quercetin nano-formulations in asthma. Moreover, we have also studied the molecular mechanisms showing the molecular encapsulation pattern of quercetin in liquid crystalline nanoparticles.

Our study, emphasizing the application of nano-quercetin in the intervention of asthma as a promising drug delivery system, would be of great interest to the formulation, biological and respiratory scientists and clinicians. Our study can form the basis for further detailed research in this field, which can help in targeting a better treatment option for asthma. We hope that you find our manuscript acceptable for publication in the Journal of Drug Delivery Science and Technology.

Kind regards, Dr Kamal Dua 
$24^{\text {th }}$ September 2019

The Editor

Journal of Drug Delivery Science \& Technology

Sub: Revision of manuscript for publication in Journal of Drug Delivery Science \& Technology

Dear Editor,

Please find enclosed our revised manuscript "Preparation, Characterization and In-vitro Efficacy of Quercetin Loaded Liquid Crystalline Nanoparticles for the Treatment of Asthma" which we would like to re-submit for consideration for publication. We are grateful to you, and that to your reviewers for providing us valuable feedback/suggestions, which we have addressed that has helped improve our manuscript. We am submitting herewith the revised manuscript which incorporates all the suggestions of the reviewers. Changes made in the revised manuscript are done using the red color mode in MS Word file along with below listed point to point clarifications to the reviewer's comments.

Best Regards

Kamal Dua/ Dinesh Kumar Chellappan

Point wise changes have been made in the revised manuscript.

We thank the Editor, Editorial team and the Reviewers for their consideration of our manuscript, and for providing valuable suggestions. We have now addressed all the comments and incorporated changes into a revised version as described in the point-by-point response below.

\section{Reviewer 1}

The manuscript entitled by Yong et al. showed the anti-inflammatory potential of the quercetin loaded liquid crystalline nanoparticles (LCN) and surface-modified liquid crystalline nanoparticles (sm-LCN) in asthma where the authors have carried out a blend of physicochemical, molecular modeling characterization and biological studies. The concept of liquid crystalline nanoparticles (LCN) and surfacemodified liquid crystalline nanoparticles is novel and emerging in the area of translational research in respiratory diseases. I recommend the manuscript to be considered for publication considering the authors can incorporate and answer the below suggestions.

1. Comment 1 (C1): There are no recent statistics provided for asthma as a disease burden. Please include in the introduction section.

Response (R1): We appreciate the reviewer comment. The statistics for disease burden for Asthma has been included in the introduction. (Page:3; line 4 to 7 ).

2. C2: Is there any particular pathway authors envisages in relation to their biological activity results, if so, please include in the discussion.

R2: We appreciate reviewer's comment. The NFאB pathway has been discussed specifically in context to our biological activity. (Page:10; line 16).

3. C3: Why the authors have selected IL- 6, IL- 8 and IL-1 $\beta$ as the potential inflammatory markers to examine in the study. Please justify.

R3: We appreciate the comment made by the reviewer. IL-6, IL- 8 and IL- $1 \beta$ has been extensively involved in pathogenesis of Asthma. The authors emphasized these inflammatory markers based on previous studies as follows: https://www.ncbi.nlm.nih.gov/pubmed/20816188, https://www.ncbi.nlm.nih.gov/pubmed/29781141 
4. C4: The authors need to check the manuscript for language consistency and grammatical errors.

R4: We appreciate reviewer's concern. The authors have checked the manuscript for grammatical errors and language consistency.

5. C5: Please ensure that all the references are correctly cited and are in accordance with the journal guidelines.

R5: The manuscript has been referenced by Endnote software as per the journal guidelines and specifications.

\section{Reviewer 2}

The manuscript entitled "Preparation, Characterization and Comparative In-vitro Efficacy of Quercetin Loaded Liquid Crystalline for the Treatment of Asthma" demonstrates the development of quercetin loaded liquid crystalline nanoparticles and surface modified liquid crystalline nanoparticles as formulations having anti-inflammatory activity in human primary bronchial epithelial cell line induced with lipopolysaccharide. The authors have covered all the important components needed for the formulation preparation and testing. However, I have a few below mentioned suggestions which authors can attend and include necessary details in the current version of their research manuscript.

1. C1: Is there any particular phenotype of asthma being targeted in the study? If so, mention the details.

R1: The study was considered to target overall population with asthma pathophysiological features but did not take into account the specific consideration for the phenotype which forms the part of our future studies.

2. C2: Introduction lacks a description of the inflammatory markers involved in the pathophysiology of asthma. The authors must include a description with the figure if possible, to provide an overview to the readers considering the authors have done the inflammatory markers assessment in their study.

R2: We appreciate reviewer's suggestion. We have included the role of inflammatory markers in the introduction section. (Page: 3; Line 7 to 11 )

3. C3: How CHI solution has been prepared, which solvent has been used please mention?

R3: The authors thank the reviewers for the feedback. The preparation of CHI solution has been included. (Page: 5 ; line: $9 \& 10$ )

4. C4: Please change morphology to surface morphology.

R4: The authors have rectified the sub-heading.

5. C5: Why authors have selected $0.5,1,2,3,6,9,12 \& 24 \mathrm{hrs}$ as the time point for in vitro release study? Please justify.

R5: The following time-points $(0.5,1,2,3,6,9,12 \& 24 \mathrm{~h})$ has been selected considering the intent to look for the prolonged/controlled release of Quercetin in the biological fluids which is the main rationale behind designing the liquid crystalline nanoparticles.

6. C6: Please be consistent with using short forms such as " $\mathrm{h}$ " for hours and $\mathrm{ml}$ or $\mathrm{mL}$ etc throughout the manuscript.

R6: The authors have made the abbreviations consistent in the manuscript.

7. C7: Fig 4C, please change IL-b which should be represented as IL-1 $\beta$.

R7: Fig 4C: IL-b has been replaced with IL-1 $\beta$.

8. C8: Authors need to do a careful reading in order to fix typographical and grammatical errors.

R8: The authors thank the review for the suggestion. Authors have read and corrected the manuscript. 


\section{Preparation, Characterization and In-vitro Efficacy of Quercetin Loaded Liquid} Crystalline Nanoparticles for the Treatment of Asthma

David Ong Cherk Yong ${ }^{1}$, Sanggetha Raja Saker ${ }^{1}$, Ridhima Wadhwa ${ }^{2,3}$, Dinesh Kumar Chellappan 4,*, Thiagarajan Madheswaran ${ }^{5}$, Jithendra Panneerselvam ${ }^{5}$, Murtaza M. Tambuwala ${ }^{6}$, Hamid A Bakshi ${ }^{6}$, Pradeep Kumar ${ }^{7}$, Viness Pillay ${ }^{7}$, Gaurav Gupta ${ }^{8}$, Brian G Oliver ${ }^{9}$, Peter Wark $^{10}$, Alan $\mathrm{Hsu}^{10}$, Philip Michael Hansbro ${ }^{2,9,10}$, Kamal Dua ${ }^{2,3,10^{*}}$, Farrukh Zeeshan ${ }^{5}$

${ }^{1}$ School of Pharmacy, International Medical University (IMU), Bukit Jalil, Kuala Lumpur, 57000, Malaysia.

${ }^{2}$ Centre for Inflammation, Centenary Institute, Sydney, NSW 2050, Australia.

${ }^{3}$ Discipline of Pharmacy, Graduate School of Health, University of Technology Sydney (UTS), Ultimo, NSW 2007, Australia.

${ }^{4}$ Department of Life Sciences, School of Pharmacy, International Medical University (IMU), Bukit Jalil, Kuala Lumpur, 57000, Malaysia.

${ }^{5}$ Department of Pharmaceutical Technology, School of Pharmacy, International Medical University (IMU), Bukit Jalil, Kuala Lumpur, 57000, Malaysia.

${ }^{6}$ School of Pharmacy and Pharmaceutical Sciences, Ulster University, Coleraine, London, United Kingdom of Great Britain and Northern Ireland.

${ }^{7}$ Wits Advanced Drug Delivery Platform Research Unit, Department of Pharmacy and Pharmacology, School of Therapeutic Sciences, Faculty of Health Sciences, University of the Witwatersrand, Johannesburg, South Africa.

${ }^{8}$ School of Pharmacy, Suresh Gyan Vihar University, Jagatpura, 302017, Mahal Road, Jaipur, India

${ }^{9}$ School of Life Sciences, Faculty of Science, University of Technology Sydney (UTS), Ultimo, NSW 2007, Australia.

${ }^{10}$ Priority Research Centre for Healthy Lungs, Hunter Medical Research Institute (HMRI) \& School of Biomedical Sciences and Pharmacy, The University of Newcastle (UoN), Callaghan, NSW 2308, Australia.

*Corresponding authors

1. Dr Dinesh Kumar Chellappan Email address: dinesh_kumar@imu.edu.my Dr Kamal Dua Email address: kamalpharmacist02@gmail.com 


\section{Abstract}

2 The present study aims to formulate quercetin loaded liquid crystalline nanoparticles (LCN) and 3 surface modified liquid crystalline nanoparticles (sm-LCN) as well as investigate their anti4 inflammatory activity in human primary bronchial epithelial cell line (BCi-NS1.1) induced with 5 lipopolysaccharide (LPS). Quercetin LCN were prepared using ultrasonication method. The

6 formulated LCNs and sm-LCNs were characterised in terms of particle size, zeta potential as well 7 as the drug encapsulation efficiency. Furthermore, their morphology and in vitro release profile 8 were also studied. In addition, the anti-inflammatory activity of quercetin LCN and sm-LCNs were 9 evaluated by measuring the concentration of pro-inflammatory markers namely interleukin (IL)$101 \beta$, IL-6 and IL-8 in BCI-NS1.1 cell lines via cytometric bead array. The molecular mechanism 11 inherent to the inclusion of quercetin into monoolein nanosystem and surface modification of the 12 nanosystem with chitosan was elucidated via molecular mechanics simulations. Quercetin LCN and sm-LCN significantly $(\mathrm{p}<0.05$ ) decreased the production of IL-1 $\beta$, IL-6 and IL-8 compared 14 to LPS only group. Encapsulation of quercetin into LCN and sm-LCN further enhanced its anti15 inflammatory activity compared to quercetin in dimethyl sulfoxide (DMSO). In addition to that, 16 quercetin LCN and sm-LCN also exhibited comparable activity to fluticasone in terms of 17 significantly $(\mathrm{p}<0.05)$ reducing the production of IL-1 $\beta$ and IL-6. Quercetin loaded LCN and sm18 LCN could be a potential therapeutic intervention for asthma as they are efficacious in suppressing 19 the production of key pro-inflammatory cytokines associated with the development of asthma. 


\section{Introduction}

Asthma is a chronic inflammatory disease involving the airways, which is often associated with narrowing of the airway structure and eventually leading to airway tissue remodeling [1]. Clinical symptoms of asthma include wheezing, coughing as well as shortness of breath. Based on the current estimates provided by the World Health Organization (WHO), around 235 million individuals suffer from asthma, thus, emerging to be a public health concern globally [2]. More than $80 \%$ of asthma deaths occur in low and lower middle-income countries. Innate immune cells, dendritic cells and epithelial cells produce various cytokines including interleukin-4 (IL-4), IL-8, IL-1 $\beta$, IL-25, IL-33, thymic stromal lymphopoietin (TSLP) resulting in Th2 differentiation, recruitment and activation, thus promote $\operatorname{IgE}$ production, eosinophil and mast cell recruitment (Figure 1). These are the important factors contributing to the pathology of asthma [3, 4]. Common treatment modalities available to relieve asthmatic symptoms and manage its exacerbations include beta-adrenergic agonist, inhaled and systemic corticosteroids. Despite corticosteroids remarkably inhibiting the cascade of inflammatory reactions pertinent in asthma, the serious long-term side effects [5] associated with its use warrant the need for a potent and safer anti-inflammatory compound $[6,7]$.

Quercetin (2-(3,4-dihydroxyphenyl)-3,5,7-trihydroxychromen-4-one) (Figure 2) is a dietary flavonoid commonly found in vegetable and fruits, including onions, apples, grapes and nuts [8]. The growing interest of researchers towards this particular flavonoid could be attributed to its various pharmacological properties such as anti-oxidant, anti-inflammatory, anti-bacterial, anti-diabetic and anti-cancer [9]. Numerous studies have also elucidated on the potential therapeutic use of quercetin in the treatment of asthma owing to its potent anti-inflammatory property. Quercetin was found to ameliorate allergic airway inflammation and hyperresponsiveness mediated via attenuation of the $\mathrm{NF}-\kappa \mathrm{B}$ signalling pathway $[10,11]$. Moreover, it also inhibits degranulation of mast cells [10], reduced the recruitment of eosinophils [12] and the levels of pro-inflammatory markers evident in allergic inflammatory diseases [13].

Although quercetin exhibits diverse pharmacological actions, its application in clinical practice is still limited. The main factor could be the poor bioavailability of this compound primarily due to low solubility in aqueous medium as well as poor intestinal absorption [14]. In order to overcome these drawbacks, quercetin can be encapsulated into a novel nanocarrier system 
such as liquid crystalline nanoparticles (LCNs). Monoolein (MO), a non-toxic amphiphilic lipid is normally used to construct these systems [15]. LCNs are formed by the dispersion of monoolein in water which self-assembles into a well-ordered bicontinuous cubic structure [16]. Surfactants such as poloxamer 407 ( $\mathrm{P}$ 407) need to be added into the LCN system so that it ensures the formation of a kinetically stable dispersion [17].

LCNs shows a great promise as an emerging drug delivery system due to their unique properties such as bioadhesive nature, higher hydrophobic drug loading capacity, sustained-release behaviour as well as their ability to improve physicochemical stability of encapsulated drugs [18, 19]. Studies have also proven that the surface modification of LCN with hydrophilic polymers such as chitosan may allow longer residence time, increased mucoadhesiveness and enhanced cellular uptake therefore, improving drug bioavailability. The rationale behind this approach is that the outer shell layer was claimed to be more important than those of the core because the surface is directly contact with body cells and fluids [3, 20-24].

Therefore, we believed that quercetin loaded LCNs and sm-LCNs were effective in treating inflammatory respiratory ailments such as asthma as well as to further enhance the antiinflammatory activity of quercetin. The main aim of this research was to formulate quercetin loaded LCNs and sm-LCNs followed by characterising them and evaluating their in vitro release profile. Finally, the effects of quercetin loaded LCNs and sm-LCNs on the secretion of proinflammatory cytokines were evaluated in immortalised human bronchial epithelial cell line (BCiNS1.1) induced with lipopolysaccharide (LPS).

\section{Materials}

Quercetin was purchased from Sisco Drug Laboratories, India; whereas, monoolein was obtained from PI Chemicals, China. Poloxamer 407, Spectra Por ${ }^{\circledR}$ dialysis membrane and fluticasone propionate were purchased from Sigma-Aldrich, USA. Immortalized human bronchial epithelial cell line, BCi-NS1.1 was acquired from R. G. Crystal (Weill Cornell Medical College, New York, USA). Bronchial Epithelial Growth Media (BEGM) and Bronchial Epithelial Basal Media (BEBM) were purchased from Lonza, USA. All other solvents and chemicals used were of analytical grade.

\subsection{Preparation of quercetin loaded LCN}


Ultrasonication method was used to prepare quercetin loaded LCNs as described previously [15].

2 The composition of quercetin loaded LCN formulation is shown in Table 1. Briefly, appropriate amounts of monoolein and quercetin were melted together in a glass vial at $45^{\circ} \mathrm{C}$. Distilled water heated to the same temperature was added into another glass vial containing P 407 and subsequently vortexed for one minute. Then, P 407 solution was transferred to the melted MO and quercetin mixture followed by vortexing for another minute. The liquid crystalline cubic phase formed in the mixture was then subjected to ultrasonication for $10 \mathrm{~min}$ using probe type sonicator

8 (Sartorius Labsonic P) at 60 amplitudes and 0.5 cycle). Blank LCN without quercetin was also 9 prepared using the same method mentioned above for comparison.

\section{Preparation of quercetin loaded sm-LCN}

$0.1 \%$ Q sm-LCN was prepared by mixing $5 \mathrm{~mL} 0.1 \% \mathrm{CHI}$ solution and $5 \mathrm{~mL}$ Q LCN. CHI solution was prepared in $50 \mathrm{mM}$ acetate buffer having $0.02 \%$ sodium azide. After that, it was subjected to magnetic stirring for $30 \mathrm{~min}$. The same process was repeated for $0.2 \% \mathrm{Q}$ sm-LCN except that it was mixed with $0.2 \%$ CHI solution [15].

\subsection{Characterization of LCN \& sm-LCN}

\subsubsection{Particle Size and Zeta Potential}

The particle size and zeta potential of the nanoparticles was evaluated using Zetasizer Nano ZS (Malvern, UK) at $25^{\circ} \mathrm{C} .0 .1 \mathrm{~mL}$ from blank LCN, Q LCN, $0.1 \%$ Q sm-LCN and $0.2 \%$ Q sm-LCN were diluted and equilibrated for 120 seconds before analysis [25-31].

\subsubsection{Surface Morphology}

The morphology of nanoparticles was studied using transmission electron microscopy (TEM).

22 Nanoparticles was diluted suitably to 50-fold and allowed to drop on a carbon-coated copper grid and fixed by vacuum drying. The anchored droplets were then exposed to TEM observation at an acceleration voltage of $100 \mathrm{kV}[15,28,32]$.

\subsubsection{Entrapment Efficiency}

The EE of the nanoformulation was evaluated by separating free drug from the nanoparticles system via centrifugation method. $1.9 \mathrm{~mL}$ of distilled water was added to dilute the $0.1 \mathrm{~mL}$ of Q LCN and Q sm-LCN. UV-vis was then performed to analyze their concentration via Shimadzu 
1 spectrophotometer at $369 \mathrm{~nm} .2 \mathrm{~mL}$ of Q LCN and Q sm-LCN were centrifuged using a benchtop

2 centrifuge (Eppendorf, Germany) at 2.5 Rcf for $15 \mathrm{~min}$. The supernatants were withdrawn

3 cautiously to remove the free quercetin and diluted with distilled water to $2 \mathrm{~mL}$. Their absorbance

4 was detected $369 \mathrm{~nm}$ using UV-Vis spectrophotometer, and the percentage of entrapment

5 efficiency was calculated according to the following equation:

$6 \% \mathrm{EE}=\left(\mathrm{D}_{\text {total }}-\mathrm{D}_{\text {free }}\right) / \mathrm{D}_{\text {total }} * 100$

$7 \quad$ Where $D_{\text {free }}$ and $D_{\text {total }}$ are the amounts of free and total quercetin in the nanoparticles.

\subsection{In vitro release study}

In vitro drug release from the nanoformulation was carried out by using Spectra/Por dialysis membrane bag. $2 \mathrm{~mL}$ of Q LCN, $0.1 \% \mathrm{Q}$ sm-LCN and $0.2 \% \mathrm{Q}$ sm-LCN was placed into separate

12 dialysis bags (MWCO: $3500 \mathrm{~g} / \mathrm{mole})$ and immersed into $25 \mathrm{~mL}$ of release medium $(0.01-\mathrm{M}$ 13 phosphate buffer, $\mathrm{pH} 7.4$ ) in falcon tube. The falcon tube was shaken at $39 \mathrm{rpm}$ at $37^{\circ} \mathrm{C}$ temperature 14 using water bath. At $0.5,1,2,3,6,9,12$ and $24 \mathrm{hrs}$ of the time points, $2 \mathrm{~mL}$ from the falcon tube 15 was removed for analysis and same amount of fresh dialysis medium was added to maintain the 16 conditions. The amount of quercetin released was measured using UV-vis spectrophotometer at $17369 \mathrm{~nm}[27,33]$.

\subsection{In vitro cell culture study}

19 Human minimally immortalized bronchial epithelial cell line BCi-NS1.1 was obtained from R. G. 20 Crystal (Weill Cornell Medical College, New York, USA), and was cultured in BEGM growth 21 media supplemented with (Lonza). Cells were then treated with Q LCN, $0.2 \%$ Q sm-LCN, blank 22 LCN, blank sm-LCN at $25 \mathrm{uM}$ for $24 \mathrm{hrs}$, and were then stimulated with LPS (100ng/mL) for $24 \mathrm{hrs}$. 23 10nM Fluticasone was used as a standard treatment. The supernatants and RNAs were then 24 harvested for further studies [25].

\subsection{Cytometric bead array}


1 The concentration of IL-1 $\beta$, IL-6 and IL-8 in the supernatants collected from the treated BCi-

2 NS1.1 cells was measured using cytometric bead array (BD Biosciences) and flow cytometry

3 (FACSCANTO II, BD Biosciences) as documented in the manufacturer's instructions [34, 35].

\subsection{Mechanistic profiling via molecular mechanics simulations}

5 Molecular mechanics simulations were carried out employing using HyperChem ${ }^{\mathrm{TM}}$ 8.0.8

6 Molecular Modelling Software (Hypercube Inc., Gainesville, FL, USA). The molecular structures

7 of chitosan (Cht; 4 glucosamine units) and quercetin (Quer) were generated employing saccharide

8 building tool and natural bond angles, respectively. For monoolein (MO), the molecular segment

9 with functional groups was generated using natural bond angles. The bimolecular structures - MO-

10 Quer and MO-Cht - were developed by parallel disposition of constituent molecules and then

11 AMBER 3 (Assisted Model Building and Energy Refinements) Force Field was applied for energy

12 minimization. Full geometrical optimization was conducted in vacuum employing the Polak-

13 Ribiere Conjugate Gradient method until an RMS gradient of $0.001 \mathrm{kcal} / \mathrm{mol}$ was reached [36].

\subsection{Statistical analysis}

15 All the values obtained were studied and performed using Graphpad Prisms 8.0. Data shown as 16 mean \pm standard deviation. One-way ANOVA tool was used to analyze the significant difference 17 between groups and a value of $\mathrm{p}<0.05$ was considered statistically significant.

\section{Results}

The mean particle size, polydispersity index (PDI) and zeta potential of the quercetin formulation were measured and summarized in table 1. The mean particle size of the LCN formulations ranged from 210.0 to $268.7 \mathrm{~nm}$ with PDI less than 0.4 for all preparations. The zeta potentials for LCN

22 formulations ranged from -14.6 to $-15.6 \mathrm{mV}$ whereas the sm-LCN ranged from 15.8 to $22.3 \mathrm{mV}$.

23 The encapsulation efficiency of quercetin loaded LCN was $99.4 \%$ which was slightly higher 24 compared to the surface modified LCNs.

25 The TEM images (Figure 3) show that both LCN and sm-LCN were spherical in shape. The 26 particle size of LCNs ranged from $100 \mathrm{~nm}$ to $300 \mathrm{~nm}$ which was consistent with the dynamic light 27 scattering. 
The release profile of quercetin LCN over 24 hours. Release profile of quercetin from LCN exhibits a prolonged manner over the longer duration of time ( Figure 4).

The BCi-NS1.1 cells were treated simultaneously with either $100 \mathrm{ng} / \mathrm{mL}$ of LPS alone or with quercetin loaded LCNs and free quercetin in DMSO solution to investigate their respective effects on the production of LPS induced pro-inflammatory markers in these cell lines. Quercetin loaded LCNs and sm-LCNs with the dose of $25 \mu \mathrm{M}$ showed a consistent trend of significantly ( $\mathrm{p}<$ 0.05 ) decreasing the concentration of pro-inflammatory cytokines namely IL-1 $\beta$, IL-6 and IL-8 compared to the positive control group (Figure 5). It is also worth noting that quercetin LCNs as well as sm-LCNs remarkably downregulated the production of IL-6, IL-8 and IL-1 $\beta$ compared to free quercetin dissolved in DMSO, therefore, exhibiting superior anti-inflammatory activity.

\section{Discussion}

\subsection{Characterization of LCN and sm-LCN}

The significant increase in particle size indicates the encapsulation of quercetin into the blank LCN. Drug encapsulation into the nanoparticles may be a result of chemical conjugation or physical entrapment [37]. Polydispersity index (PDI) represents the size distribution and uniformity of the nanoparticles formed [38]. PDI values greater than 0.3 indicates a broad size distribution. Ideally, a narrow size distribution identified with PDI values lesser than 0.3 is preferred [38]. The slightly higher polydispersity index of quercetin loaded LCNs may be attributed to the coexistence of cubic liquid crystalline nanoparticles with other types of vesicles in the dispersion as previously reported $[39,40]$. It was observed that the mean particle size for sm-LCN was significantly larger than $\mathrm{LCN}$ with regards to the amount of $\mathrm{CHI}$ used to modify the surface. When $0.1 \% \mathrm{CHI}$ was used, the particle size observed was $236.1 \mathrm{~nm}$ whereas $0.2 \% \mathrm{CHI}$ used has shown to increase the particle size to $268.7 \mathrm{~nm}$. The size increased in proportion to the quantity of $\mathrm{CHI}$ used proves that the increment was attributed to the surface coating of $\mathrm{CHI}$ on LCN [15].

The positive reading in zeta potential further indicates that the surface of LCN (negatively charged) have been successfully coated by the protonated amino group from CHI (13). Generally, zeta potential values above $\pm 25 \mathrm{mV}$ are required to form a stable dispersion between particles and reduce their aggregation [41]. P 407 aided in stabilising the LCN dispersion as demonstrated 
previously [42]. It is presumed that the hydrophobic part of the polypropylene oxide chain adheres to the surface of the cubic nanoparticles whereas the hydrophilic portion of the chain extends out to the surrounding environment to provide steric shielding, therefore, avoiding agglomeration [43]. The negatively charged zeta potential could be ascribed to the subtle amount of oleic acid released by monoolein resulting in anionic behavior of the dispersion caused by adsorption of hydroxyl ions on the surface of the LCNs $[41,43]$.

TEM images further confirmed the results from the Zetasizer, showing that the particles were spherical in shape and size less than $300 \mathrm{~nm}$, which is quite similar to other reported studies $[15,18,42,44]$. The dense black core surrounding the particle was due to the existence of CHI coating indicating that the surface of LCN has been modified.

Both LCN and sm-LCN have exhibited a high entrapment efficiency whereby $>97 \%$ of drug was successfully entrapped. This indicates that most of the drug has been encapsulated regardless the surface modification of the nanoparticle. However other studies have successfully encapsulated their drug as high as $99 \%$ in $\operatorname{LCN}[15,18,41]$. Nevertheless, the entrapment efficiency is still considered high when compared to other nanocarriers such as poly lactide-coglycolic acid (PLGA) polymer-based nanoparticles where Anwar et al reported that only $86 \%$ of quercetin was encapsulated [45].

\subsection{In vitro release study}

The result has shown that quercetin drug was able to release in a sustained release manner when incorporated into the LCN and by modifying the surface with $\mathrm{CHI}$ further prolonged the drug release. This sustained release of quercetin could be attributed to the structure of the LCN. The higher proportion of lipids in the LCN not only allows hydrophobic drug to bind strongly to its inner structure, but also provides a larger surface area for the drugs to be incorporated. As a result, high entrapment efficiency of the drug can be achieved. Yoo et al., also showed that the release of the drug from LCN has sustained as long as 15 days [18].

\subsection{In vitro evaluation anti-inflammatory activity of quercetin loaded $\mathrm{LCN}$ and} sm-LCN on immortalised human bronchial epithelial cell line (BCi-NS1.1) 
3 In the present study, quercetin loaded LCNs effectively decreased the concentration of pro4 inflammatory cytokines namely IL-1 $\beta$, IL-6 and IL-8. These effects exhibited by quercetin LCN 5 was in consistent with previous studies evaluating the anti-inflammatory activity of quercetin on 6 different cell lines [11, 13, 46-48]. Zhang et al., proved that quercetin remarkably suppressed the secretion of IL-6 and IL-1 $\beta$ in LPS-stimulated human peripheral blood mononuclear cells (PBMCs) [47]. Another research demonstrated that the treatment of quercetin notably reduced the gene expression and release of IL-8, IL-6 as well as IL-1 $\beta$ in human mast cells (HMC-1) induced by phorbol 12-myristate 13-acetate and calcium ionophore A23187 [11]. Although the underlying mechanism was not investigated in this present study, it is well-established from previous studies that quercetin attenuates the activation of NF- $\kappa \mathrm{B}$ hence, downregulating the production of proinflammatory cytokines [11, 46, 47]. NF-kB signaling pathway plays a crucial role in the pathogenesis of asthma as it modulates the gene expression of pro-inflammatory mediators including chemokines, cytokines and cell adhesion molecules that lead to the infiltration of immune cells such as neutrophils and eosinophils in the airway $[49,50]$. improving its anti-inflammatory activity. The enhanced anti-inflammatory effects of quercetin LCNs could be primarily attributed to the characteristics of the LCN itself, whereby it is bioadhesive in nature and improves membrane permeation leading to an increased interaction with the bronchial epithelial cells $[41,51]$. The lipid bilayer of LCNs is akin to the epithelial cell absorption [51, 52]. Furthermore, findings from numerous studies also elucidated that LCNs promote greater cellular uptake and internalisation into cells via endocytosis enabling them to be diffused throughout the cytoplasm $[18,41,52,53]$. On the other hand, quercetin itself could have only remained in the cell membrane due to its high lipophilicity allowing it to be easily incorporated into the cell membrane.

The findings observed in our study has demonstrated that quercetin loaded sm-LCN with the dose of $25 \mu \mathrm{M}$ significantly $(\mathrm{p}<0.05)$ decreased the pro-inflammatory markers when compared 
to the free quercetin drug in DMSO. This indicates that sm-LCN is able to enhance the antiinflammatory effect of quercetin when entrapped in the nanocarrier.

There is also a noticeable reduction in the expression of all the pro-inflammatory cytokines which was observed on the blank LCN \& sm-LCN. This suggests that the empty vehicle itself can slightly reduce the inflammation. We suspect that one of the components in the LCN formulation might contributed and it was monoolein. However, only one study has confirmed this and Ali et al reported that monoolein possess anti-inflammatory property that inhibits the production of proinflammatory markers in LPS stimulated bone marrow-derived dendritic cell (BMDC) via NF- $\kappa B$ and MAPK pathways [54].

\section{Evaluation of in vitro anti-inflammatory activity of quercetin loaded $L C N$ and $s m-L C N$ in} comparison to fluticasone

Fluticasone, an FDA approved drug to treat asthma was used as a standard to further establish the anti-inflammatory activity of quercetin LCNs. Quercetin LCNs were comparable to fluticasone in terms of reducing the levels of IL-1 $\beta$ and IL- 6 however, fluticasone was much more effective in decreasing the production of IL-8. In this existing study, the BCi-NS1.1 cell lines were only treated with quercetin LCNs at the dose of $25 \mu \mathrm{M}$. Quercetin was able to reduce the levels of proinflammatory cytokines in a dose-dependent manner up to a $100 \mu \mathrm{M}$ as described in the literature [47]. Therefore, the finding suggests the potential of quercetin LCNs to decrease the levels proinflammatory cytokines to a greater extent comparable to fluticasone at a higher dose.

\subsection{Molecular mechanics simulations}

The molecular simulations performed in this research focused on two aspects: a) encapsulation or incorporation of quercetin within a monoolein matrix, and b) surface modification of the monoolein matrix with chitosan. The geometrical iterations representing the above bimolecular complexes are shown in Figure 5 while the related energetic molecular attributes are depicted in Tables 2 and 3, respectively. In case of MO-Quer; the formation of molecular complex was accompanied by a total energy stabilization of $15.894 \mathrm{kcal} / \mathrm{mol}$. Interestingly, the total energy of the MO-Quer complex was less than the individual constituent molecules. The incorporation of Quer in MO was evident from the fact that all bonding (bond, angle, and dihedral) and non-bonding (van der waals forces and H-bonding) energy terms contributed to the geometrical stabilization of 
the complex. The major stabilization was provided by the van der Waals function which brought

2 the molecules in close vicinity and hence led to the formation of - $\mathrm{OH}$...HO- bonds between the molecules (Table 2, Figure 5). For the MO-Cht molecular complex; an energy stabilization of $10.336 \mathrm{kcal} / \mathrm{mol}$ was observed. In line with MO-Quer, the total energy of the MO-Cht complex was

5 less than the individual molecular components. The energy stabilization in this case was mainly

6 due to non-bonding interactions (van der Waals forces and electrostatic interactions) while the

7 bonding interactions (bond and dihedral energies) destabilized the complex. Such destabilization

8 is due to torsional strain experienced by the molecules while interacting within the van der Waals

9 space. Additionally, both inter- and intra-molecular H-bonding was observed in the MO-Cht complex further confirming its favourable formation and conformation. The above discussion justifies the selection and successful incorporation of quercetin in and chitosan surface modification of the monoolein nanosystem.

\section{Conclusions}

Quercetin was successfully encapsulated into liquid crystalline nanoparticles revealing their sustained release behaviour. Moreover, quercetin LCN and sm-LCN were effective in reducing the levels of pro-inflammatory mediators such as IL-1 $\beta$, IL-6 and IL-8 whereby encapsulating quercetin into LCN further enhanced its anti-inflammatory activity. This demonstrates that the quercetin LCN could be employed as a potential novel drug delivery for the treatment of asthma. This mode will overcome the issues associated with quercetin such as the low solubility, poor bioavailability and short half-life. The findings of this present study advocate the translation into other associated pulmonary inflammatory diseases such as chronic obstructive pulmonary disorder (COPD) as well as lung cancer which will help in providing a new direction to the pulmonary clinics.

\section{References}

1. Tanaka, T. and R. Takahashi, Flavonoids and asthma. Nutrients, 2013. 5(6): p. 2128-2143.

Asthma. Facts about asthma 2017 [cited 20196 September]; Available from: https://www.who.int/news-room/fact-sheets/detail/asthma.

3. Dua, K., et al., Increasing complexity and interactions of oxidative stress in chronic respiratory diseases: An emerging need for novel drug delivery systems. Chemico-biological interactions, 2018. 
4. Wadhwa, R., et al., Nanoparticle-Based Drug Delivery for Chronic Obstructive Pulmonary Disorder Elsevier. p. 59-73.

5. $\quad$ Pandya, D., A. Puttanna, and V. Balagopal, Suppl 1: M2: Systemic Effects of Inhaled Corticosteroids: An Overview. The open respiratory medicine journal, 2014. 8: p. 59.

6. Hansbro, P.M., et al., Mechanisms and treatments for severe, steroid-resistant allergic airway disease and asthma. Immunological reviews, 2017. 278(1): p. 41-62.

7. Dua, K., N.G. Hansbro, and P.M. Hansbro, Steroid resistance and concomitant respiratory infections: A challenging battle in pulmonary clinic. EXCLI journal, 2017. 16: p. 981.

8. Wang, W., et al., The biological activities, chemical stability, metabolism and delivery systems of quercetin: A review. Trends in Food Science \& Technology, 2016. 56: p. 21-38.

9. D'Andrea, G., Quercetin: a flavonol with multifaceted therapeutic applications? Fitoterapia, 2015. 106: p. 256-271.

10. Cruz, E., et al., Kalanchoe pinnata inhibits mast cell activation and prevents allergic airway disease. Phytomedicine, 2012. 19(2): p. 115-121.

11. Park, H.-H., et al., Flavonoids inhibit histamine release and expression of proinflammatory cytokines in mast cells. Archives of pharmacal research, 2008. 31(10): p. 1303.

12. Oliveira, T.T., et al., Potential therapeutic effect of Allium cepa L. and quercetin in a murine model of Blomia tropicalis induced asthma. DARU Journal of Pharmaceutical Sciences, 2015. 23(1): p. 18.

13. Takashima, K., et al., Protective effects of intratracheally administered quercetin on lipopolysaccharide-induced acute lung injury. Respiratory research, 2014. 15(1): p. 150.

14. Sharma, S., et al., Rutin: therapeutic potential and recent advances in drug delivery. Expert opinion on investigational drugs, 2013. 22(8): p. 1063-1079.

15. Madheswaran, T., et al., Enhanced skin permeation of 5a-reductase inhibitors entrapped into surface-modified liquid crystalline nanoparticles. Archives of pharmacal research, 2015. 38(4): p. 534-542.

16. Mulet, X., B.J. Boyd, and C.J. Drummond, Advances in drug delivery and medical imaging using colloidal lyotropic liquid crystalline dispersions. Journal of colloid and interface science, 2013. 393 : p. 1-20.

17. Madheswaran, T., et al., Enhanced topical delivery of finasteride using glyceryl monooleate-based liquid crystalline nanoparticles stabilized by cremophor surfactants. AAPS PharmSciTech, 2014. 15(1): p. 44-51.

18. Baskaran, R., et al., Entrapment of curcumin into monoolein-based liquid crystalline nanoparticle dispersion for enhancement of stability and anticancer activity. International journal of nanomedicine, 2014. 9: p. 3119.

19. Dua, K., et al., Nanotechnology: Advancing the translational respiratory research. Interventional Medicine and Applied Science, 2017. 9(1): p. 39-41.

20. Choi, S.W., W.S. Kim, and J.H. Kim, Surface-functionalized nanoparticles for controlled drug delivery. Methods Mol Biol, 2005. 303: p. 121-31.

21. Al-Nemrawi, N.K., A.R. Okour, and R.H. Dave, Surface modification of PLGA nanoparticles using chitosan: Effect of molecular weight, concentration, and degree of deacetylation. Advances in Polymer Technology, 2018. 37(8): p. 3066-3075.

22. Dua, K., et al., Nanotechnology in drug delivery gaining new perspectives in respiratory diseases. Panminerva medica, 2018. 60(3): p. 135.

23. Dua, K., et al., Multi-drug resistant Mycobacterium tuberculosis \& oxidative stress complexity: Emerging need for novel drug delivery approaches. Biomedicine \& Pharmacotherapy, 2018. 107: p. $1218-1229$. 
24. Mehta, M., et al., Interactions with the macrophages: An emerging targeted approach using novel drug delivery systems in respiratory diseases. Chemico-biological interactions, 2019.

25. Ng, Z.Y., et al., Assessing the potential of liposomes loaded with curcumin as a therapeutic intervention in asthma. Colloids and Surfaces B: Biointerfaces, 2018. 172: p. 51-59.

26. Das, P., et al., Potential therapeutic activity of Phlogacanthus thyrsiformis Hardow (Mabb) flower extract and its biofabricated silver nanoparticles against chemically induced urolithiasis in male Wistar rats. International journal of biological macromolecules, 2017. 103: p. 621-629.

27. Madan, J.R., et al., Formulation, Characterization and In-vitro Evaluation of Fast Dissolv ing Tablets Containing Gliclazide Hydrotropic Solid Dispersions. Recent Pat Drug Deliv Formul, 2017. 11(2): p. 147-154.

28. Chellappan, D.K., et al., Formulation and characterization of glibenclamide and quercetin-loaded chitosan nanogels targeting skin permeation. Therapeutic delivery, 2019. 10(5): p. 281-293.

29. Tambuwala, M.M., et al., Albumin nano-encapsulation of caffeic acid phenethyl ester and piceatannol potentiated its ability to modulate HIF and NF-kB pathways and improves therapeutic outcome in experimental colitis. Drug delivery and translational research, 2019. 9(1): p. 14-24.

30. Abdelkader, D.H., et al., Enhanced cutaneous wound healing in rats following topical delivery of insulin-loaded nanoparticles embedded in poly (vinyl alcohol)-borate hydrogels. Drug delivery and translational research, 2018. 8(5): p. 1053-1065.

31. Khan, M.N., et al., Polymeric nano-encapsulation of curcumin enhances its anti-cancer activity in breast (MDA-MB231) and lung (A549) cancer cells through reduction in expression of HIF-1a and nuclear p65 (REL A). Current drug delivery, 2018. 15(2): p. 286-295.

32. Das, P., et al., Antibacterial and In Vitro Growth Inhibition Study of Struvite Urinary Stones Using Oxalis corniculata Linn. Leaf Extract and its Biofabricated Silver Nanoparticles. Recent patents on drug delivery \& formulation, 2018. 12(3): p. 170-178.

33. Malipeddi, V.R., R. Awasthi, and K. Dua, Formulation and evaluation of controlled release ethylcellulose and polyethylene glycol microspheres containing metoprolol tartrate. Interventional Medicine and Applied Science, 2016. 8(2): p. 60-67.

34. Hsu, A.C., et al., MicroRNA-125a and-b inhibit A20 and MAVS to promote inflammation and impair antiviral response in COPD. JCl insight, 2017. 2(7).

35. Baines, K.J., et al., Novel immune genes associated with excessive inflammatory and antiviral responses to rhinovirus in COPD. Respiratory research, 2013. 14(1): p. 15.

36. Kumar, P., Y.E. Choonara, and V. Pillay, In silico analytico-mathematical interpretation of biopolymeric assemblies: Quantification of energy surfaces and molecular attributes via atomistic simulations. Bioengineering \& translational medicine, 2018. 3(3): p. 222-231.

37. He, X., et al., Curcumin-loaded lipid cubic liquid crystalline nanoparticles: preparation, optimization, physicochemical properties and oral absorption. Journal of nanoscience and nanotechnology, 2015. 15(8): p. 5559-5565.

38. Danaei, M., et al., Impact of particle size and polydispersity index on the clinical applications of lipidic nanocarrier systems. Pharmaceutics, 2018. 10(2): p. 57.

39. Nasr, M., M.K. Ghorab, and A. Abdelazem, In vitro and in vivo evaluation of cubosomes containing 5-fluorouracil for liver targeting. Acta pharmaceutica sinica B, 2015. 5(1): p. 79-88.

40. Nanjwade, B.K., et al., Development of cuboidal nanomedicine by nanotechnology. Austin J Nanomed Nanotechnol, 2014. 2(4): p. 1023.

41. Freag, M.S., et al., Stealth, biocompatible monoolein-based lyotropic liquid crystalline nanoparticles for enhanced aloe-emodin delivery to breast cancer cells: in vitro and in vivo studies. International journal of nanomedicine, 2016. 11: p. 4799.

42. Madheswaran, T., et al., Design and in vitro evaluation of finasteride-loaded liquid crystalline nanoparticles for topical delivery. AAPS PharmSciTech, 2013. 14(1): p. 45-52. 
43. Verma, P. and M. Ahuja, Cubic liquid crystalline nanoparticles: optimization and evaluation for ocular delivery of tropicamide. Drug delivery, 2016. 23(8): p. 3043-3054.

44. Thapa, R.K., et al., Preparation, characterization, and release study of tacrolimus-loaded liquid crystalline nanoparticles. Journal of Dispersion Science and Technology, 2013. 34(1): p. 72-77.

45. Anwer, M.K., et al., Development and evaluation of PLGA polymer based nanoparticles of quercetin. Int J Biol Macromol, 2016. 92: p. 213-219.

46. Min, Y.D., et al., Quercetin inhibits expression of inflammatory cytokines through attenuation of NF-kappaB and p38 MAPK in HMC-1 human mast cell line. Inflamm Res, 2007. 56(5): p. 210-5.

47. Zhang, M., et al., Quercetin ameliorates LPS-induced inflammation in human peripheral blood mononuclear cells by inhibition of the TLR2-NF-kappaB pathway. Genet Mol Res, 2016. 15(2).

48. Weng, Z., et al., Quercetin is more effective than cromolyn in blocking human mast cell cytokine release and inhibits contact dermatitis and photosensitivity in humans. PLoS One, 2012. 7(3): p. e33805.

49. Schuliga, M., NF-kappaB Signaling in Chronic Inflammatory Airway Disease. Biomolecules, 2015. 5(3): p. 1266-83.

50. Starkey, M., et al., 175: Interleukin-13 predisposes mice to more severe influenza infection by suppressing interferon responses and activating microRNA-21/PI3K. Cytokine, 2014. 70(1): p. 70.

51. Jin, X., et al., A nanostructured liquid crystalline formulation of 20 (S)-protopanaxadiol with improved oral absorption. Fitoterapia, 2013. 84: p. 64-71.

52. Luo, Q., et al., A novel glyceryl monoolein-bearing cubosomes for gambogenic acid: preparation, cytotoxicity and intracellular uptake. International journal of pharmaceutics, 2015. 493(1-2): p. 30-39.

53. Abdel-Bar, H.M. and R.A. el Basset Sanad, Endocytic pathways of optimized resveratrol cubosomes capturing into human hepatoma cells. Biomedicine \& Pharmacotherapy, 2017. 93: p. 561-569.

54. Ali, I., et al., Monoolein, isolated from Ishige sinicola, inhibits lipopolysaccharide-induced inflammatory response by attenuating mitogen-activated protein kinase and NF-KB pathways. Food science and biotechnology, 2017. 26(2): p. 507-511. 


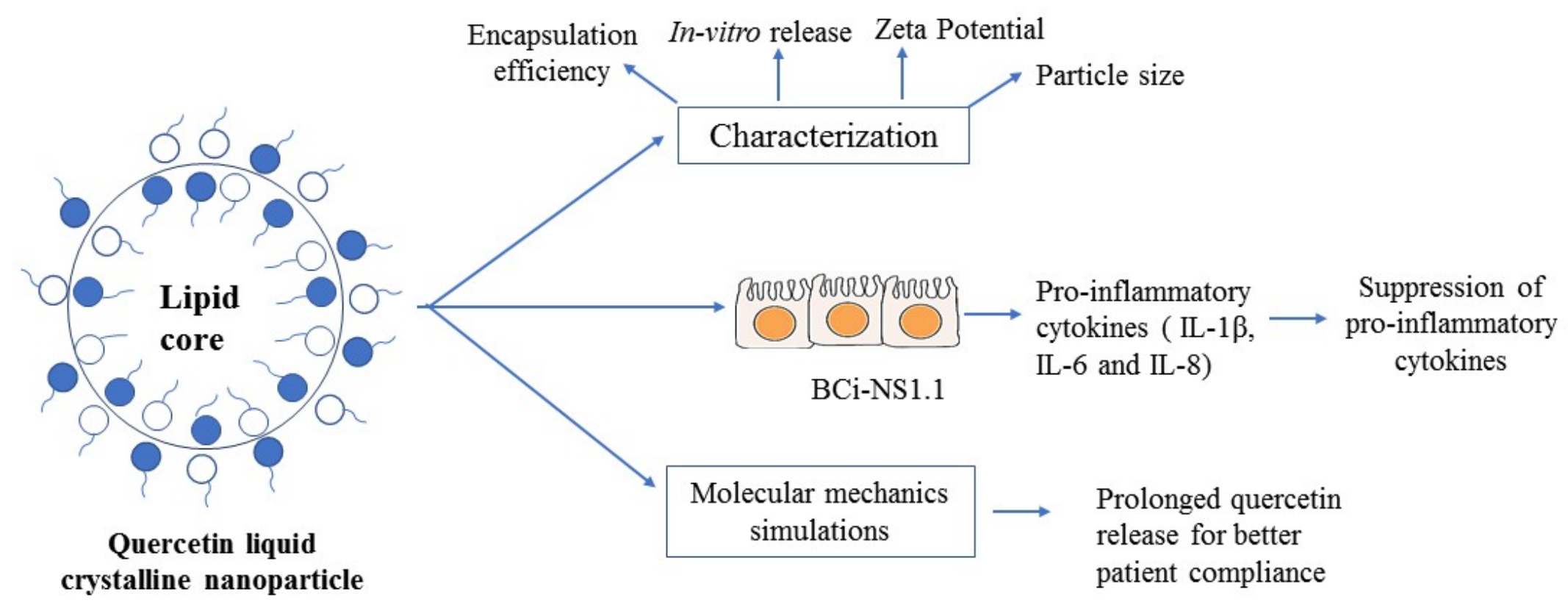




\section{Preparation, Characterization and In-vitro Efficacy of Quercetin Loaded Liquid Crystalline Nanoparticles for the Treatment of Asthma}

David Ong Cherk Yong ${ }^{1}$, Sanggetha Raja Saker ${ }^{1}$, Dinesh Kumar Chellappan,*, Thiagarajan

Madheswaran ${ }^{3}$, Jithendra Panneerselvam ${ }^{3}$, Ridhima Wadhwa ${ }^{4,5}$, Pradeep Kumar ${ }^{6}$, Viness Pillay ${ }^{6}$, Gaurav Gupta ${ }^{7}$, Brian G Oliver ${ }^{8}$, Peter Wark ${ }^{9}$, Alan Hsu ${ }^{9}$, Philip Michael Hansbro ${ }^{5,8,9}$, Kamal Dua $^{4,5,9^{*}}$, Farrukh Zeeshan ${ }^{3}$

${ }^{1}$ School of Pharmacy, International Medical University (IMU), Bukit Jalil, Kuala Lumpur, 57000, Malaysia.

${ }^{2}$ Department of Life Sciences, School of Pharmacy, International Medical University (IMU), Bukit Jalil, Kuala Lumpur, 57000, Malaysia.

${ }^{3}$ Department of Pharmaceutical Technology, School of Pharmacy, International Medical University (IMU), Bukit Jalil, Kuala Lumpur, 57000, Malaysia.

${ }^{4}$ Discipline of Pharmacy, Graduate School of Health, University of Technology Sydney (UTS), Ultimo, NSW 2007, Australia.

${ }^{5}$ Centre for Inflammation, Centenary Institute, Sydney, NSW 2050, Australia.

${ }^{6}$ Wits Advanced Drug Delivery Platform Research Unit, Department of Pharmacy and Pharmacology, School of Therapeutic Sciences, Faculty of Health Sciences, University of the Witwatersrand, Johannesburg, South Africa.

${ }^{7}$ School of Pharmacy, Suresh Gyan Vihar University, Jagatpura, 302017, Mahal Road, Jaipur, India

${ }^{8}$ School of Life Sciences, Faculty of Science, University of Technology Sydney (UTS), Ultimo, NSW 2007, Australia.

${ }^{9}$ Priority Research Centre for Healthy Lungs, Hunter Medical Research Institute (HMRI) \& School of Biomedical Sciences and Pharmacy, The University of Newcastle (UoN), Callaghan, NSW 2308, Australia.

*Corresponding authors

1. Dr Dinesh Kumar Chellappan Email address: dinesh kumar@imu.edu.my 
2. Dr Kamal Dua

Email address: kamalpharmacist02@gmail.com 



\begin{abstract}
The present study aims to formulate quercetin loaded liquid crystalline nanoparticles (LCN) and surface modified liquid crystalline nanoparticles (sm-LCN) as well as investigate their antiinflammatory activity in human primary bronchial epithelial cell line (BCi-NS1.1) induced with lipopolysaccharide (LPS). Quercetin LCN were prepared using ultrasonication method. The formulated LCNs and sm-LCNs were characterised in terms of particle size, zeta potential as well as the drug encapsulation efficiency. Furthermore, their morphology and in vitro release profile were also studied. In addition, the anti-inflammatory activity of quercetin LCN and smLCNs were evaluated by measuring the concentration of pro-inflammatory markers namely interleukin (IL)-1 $\beta$, IL-6 and IL-8 in BCI-NS1.1 cell lines via cytometric bead array. The molecular mechanism inherent to the inclusion of quercetin into monoolein nanosystem and surface modification of the nanosystem with chitosan was elucidated via molecular mechanics simulations. Quercetin LCN and sm-LCN significantly $(\mathrm{p}<0.05)$ decreased the production of IL-1 $\beta$, IL-6 and IL-8 compared to LPS only group. Encapsulation of quercetin into LCN and smLCN further enhanced its anti-inflammatory activity compared to quercetin in dimethyl sulfoxide (DMSO). In addition to that, quercetin LCN and sm-LCN also exhibited comparable activity to fluticasone in terms of significantly $(\mathrm{p}<0.05)$ reducing the production of IL-1 $\beta$ and IL-6. Quercetin loaded LCN and sm-LCN could be a potential therapeutic intervention for asthma as they are efficacious in suppressing the production of key pro-inflammatory cytokines associated with the development of asthma.
\end{abstract}

Keywords: Quercetin; Liquid crystalline nanoparticles; Asthma; Inflammation; BCi-NS1.1 


\section{Introduction}

Asthma is a chronic inflammatory disease involving the airways, which is often associated with narrowing of the airway structure and eventually leading to airway tissue remodelling [1]. Clinical symptoms of asthma include wheezing, coughing as well as shortness of breath. Based on the current estimates provided by the World Health Organization (WHO), around 235 million individuals suffer from asthma, thus, emerging to be a public health concern globally [2]. Common treatment modalities available to relieve asthmatic symptoms and manage its exacerbations include beta-adrenergic agonist, inhaled and systemic corticosteroids. Despite corticosteroids remarkably inhibiting the cascade of inflammatory reactions pertinent in asthma, the serious long-term side effects [3] associated with its use warrant the need for a potent and safer anti-inflammatory compound $[4,5]$.

Quercetin (2-(3,4-dihydroxyphenyl)-3,5,7-trihydroxychromen-4-one) (Figure 1) is a dietary flavonoid commonly found in vegetable and fruits, including onions, apples, grapes and nuts [6]. The growing interest of researchers towards this particular flavonoid could be attributed to its various pharmacological properties such as anti-oxidant, anti-inflammatory, anti-bacterial, anti-diabetic and anti-cancer [7]. Numerous studies have also elucidated on the potential therapeutic use of quercetin in the treatment of asthma owing to its potent anti-inflammatory property. Quercetin was found to ameliorate allergic airway inflammation and hyperresponsiveness mediated via attenuation of the $\mathrm{NF}-\kappa \mathrm{B}$ signalling pathway [8,9]. Moreover, it also inhibits degranulation of mast cells [8], reduced the recruitment of eosinophils [10] and the levels of pro-inflammatory markers evident in allergic inflammatory diseases [11].

Although quercetin exhibits diverse pharmacological actions, its application in clinical practice is still limited. The main factor could be the poor bioavailability of this compound primarily due to low solubility in aqueous medium as well as poor intestinal absorption [12]. In order to overcome these drawbacks, quercetin can be encapsulated into a novel nanocarrier system such as liquid crystalline nanoparticles (LCNs). Monoolein (MO), a non-toxic amphiphilic lipid is normally used to construct these systems [13]. LCNs are formed by the dispersion of monoolein in water which self-assembles into a well-ordered bicontinuous cubic structure [14]. Surfactants such as poloxamer 407 (P 407) need to be added into the LCN system so that it ensures the formation of a kinetically stable dispersion [15]. 
LCNs shows a great promise as an emerging drug delivery system due to their unique properties such as bioadhesive nature, higher hydrophobic drug loading capacity, sustainedrelease behaviour as well as their ability to improve physicochemical stability of encapsulated drugs $[16,17]$. Studies have also proven that the surface modification of LCN with hydrophilic polymers such as chitosan may allow longer residence time, increased mucoadhesiveness and enhanced cellular uptake therefore, improving drug bioavailability. The rationale behind this approach is that the outer shell layer was claimed to be more important than those of the core because the surface is directly contact with body cells and fluids [18-23].

Therefore, we believed that quercetin loaded LCNs and sm-LCNs were effective in treating inflammatory respiratory ailments such as asthma as well as to further enhance the antiinflammatory activity of quercetin. The main aim of this research was to formulate quercetin loaded LCNs and sm-LCNs followed by characterising them and evaluating their in vitro release profile. Finally, the effects of quercetin loaded LCNs and sm-LCNs on the secretion of proinflammatory cytokines were evaluated in immortalised human bronchial epithelial cell line (BCi-NS1.1) induced with lipopolysaccharide (LPS).

\section{Materials}

Quercetin was purchased from Sisco Drug Laboratories, India; whereas, monoolein was obtained from PI Chemicals, China. Poloxamer 407, Spectra Por ${ }^{\circledR}$ dialysis membrane and fluticasone propionate were purchased from Sigma-Aldrich, USA. Immortalized human bronchial epithelial cell line, BCi-NS1.1 was acquired from R. G. Crystal (Weill Cornell Medical College, New York, USA). Bronchial Epithelial Growth Media (BEGM) and Bronchial Epithelial Basal Media (BEBM) were purchased from Lonza, USA. All other solvents and chemicals used were of analytical grade.

\subsection{Preparation of quercetin loaded LCN}

Ultrasonication method was used to prepare quercetin loaded LCNs as described previously [13]. The composition of quercetin loaded LCN formulation is shown in Table 1. Briefly, appropriate amounts of monoolein and quercetin were melted together in a glass vial at $45^{\circ} \mathrm{C}$. Distilled water heated to the same temperature was added into another glass vial containing P 407 and subsequently vortexed for one minute. Then, P 407 solution was transferred to the melted MO 
and quercetin mixture followed by vortexing for another minute. The liquid crystalline cubic phase formed in the mixture was then subjected to ultrasonication for 10 minutes using probe type sonicator (Sartorius Labsonic P) at 60 amplitudes and 0.5 cycle. Blank LCN without quercetin was also prepared using the same method mentioned above for comparison.

\subsection{Preparation of quercetin loaded sm-LCN}

$0.1 \%$ Q sm-LCN was prepared by mixing $5 \mathrm{ml} 0.1 \%$ CHI solution and $5 \mathrm{ml}$ Q LCN. After that, it was subjected to magnetic stirring for $30 \mathrm{~min}$. The same process was repeated for $0.2 \% \mathrm{Q}$ smLCN except that it was mixed with $0.2 \% \mathrm{CHI}$ solution [13].

\subsection{Characterization of LCN \& sm-LCN}

\subsubsection{Particle Size and Zeta Potential}

The particle size and zeta potential of the nanoparticles was evaluated using Zetasizer Nano ZS (Malvern, UK) at $25^{\circ} \mathrm{C} .0 .1 \mathrm{~mL}$ from blank LCN, Q LCN, 0.1\% Q sm-LCN and $0.2 \%$ Q sm-LCN were diluted and equilibrated for 120 seconds before analysis [24-30].

\subsubsection{Morphology}

The morphology of nanoparticles was studied using transmission electron microscopy (TEM). Nanoparticles was diluted suitably to 50-fold and allowed to drop on a carbon-coated copper grid and fixed by vacuum drying. The anchored droplets were then exposed to TEM observation at an acceleration voltage of $100 \mathrm{kV}[13,27,31]$.

\subsubsection{Entrapment Efficiency}

The EE of the nanoformulation was evaluated by separating free drug from the nanoparticles system via centrifugation method. $1.9 \mathrm{~mL}$ of distilled water was added to dilute the $0.1 \mathrm{~mL}$ of $\mathrm{Q}$ LCN and Q sm-LCN. UV-vis was then performed to analyze their concentration via Shimadzu spectrophotometer at $369 \mathrm{~nm} .2 \mathrm{~mL}$ of Q LCN and Q sm-LCN were centrifuged using a benchtop centrifuge (Eppendorf, Germany) at 2.5 Rcf for 15 minutes. The supernatants were withdrawn cautiously to remove the free quercetin and diluted with distilled water to $2 \mathrm{~mL}$. Their absorbance was detected $369 \mathrm{~nm}$ using UV-Vis spectrophotometer, and the percentage of entrapment efficiency was calculated according to the following equation: 
$\% \mathrm{EE}=\left(\mathrm{D} \_\right.$total-D_free $) / \mathrm{D} \_$total $* 100$

Where Dfree and Dtotal are the amounts of free and total quercetin in the nanoparticles.

\subsection{In vitro release study}

In vitro drug release from the nanoformulation was carried out by using Spectra/Por dialysis membrane bag. $2 \mathrm{ml}$ of Q LCN, $0.1 \% \mathrm{Q}$ sm-LCN and $0.2 \% \mathrm{Q}$ sm-LCN was placed into separate dialysis bags (MWCO: $3500 \mathrm{~g} / \mathrm{mole}$ ) and immersed into $25 \mathrm{ml}$ of release medium (0.01-M phosphate buffer, $\mathrm{pH}$ 7.4) in falcon tube. The falcon tube was shaken at $39 \mathrm{rpm}$ at $37 \mathrm{oC}$ temperature using water bath. At $0.5,1,2,3,6,9,12$ and 24 hours of the time points, $2 \mathrm{ml}$ from the falcon tube was removed for analysis and same amount of fresh dialysis medium was added to maintain the conditions. The amount of quercetin released was measured using UV-vis spectrophotometer at $369 \mathrm{~nm}[26,32]$.

\subsection{In vitro cell culture study}

Human minimally immortalized bronchial epithelial cell line BCi-NS1.1 was obtained from R. G. Crystal (Weill Cornell Medical College, New York, USA), and was cultured in BEGM growth media supplemented with (Lonza). Cells were then treated with Q LCN, 0.2\% Q smLCN, blank LCN, blank sm-LCN at $25 \mathrm{uM}$ for $24 \mathrm{hrs}$, and were then stimulated with LPS $(100 \mathrm{ng} / \mathrm{mL})$ for $24 \mathrm{hr}$. $10 \mathrm{nM}$ Fluticasone was used as a standard treatment. The supernatants and RNAs were then harvested for further studies [24].

\subsection{Cytometric bead array}

The concentration of IL-1 $\beta$, IL- 6 and IL- 8 in the supernatants collected from the treated BCiNS1.1 cells was measured using cytometric bead array (BD Biosciences) and flow cytometry (FACSCANTO II, BD Biosciences) as documented in the manufacturer's instructions [33, 34].

\subsection{Mechanistic profiling via molecular mechanics simulations}

Molecular mechanics simulations were carried out employing using HyperChem ${ }^{\mathrm{TM}}$ 8.0.8 Molecular Modelling Software (Hypercube Inc., Gainesville, FL, USA). The molecular structures of chitosan (Cht; 4 glucosamine units) and quercetin (Quer) were generated employing 
saccharide building tool and natural bond angles, respectively. For monoolein (MO), the molecular segment with functional groups was generated using natural bond angles. The bimolecular structures - MO-Quer and MO-Cht - were developed by parallel disposition of constituent molecules and then AMBER 3 (Assisted Model Building and Energy Refinements) Force Field was applied for energy minimization. Full geometrical optimization was conducted in vacuum employing the Polak-Ribiere Conjugate Gradient method until an RMS gradient of $0.001 \mathrm{kcal} / \mathrm{mol}$ was reached [35].

\subsection{Statistical analysis}

All the values obtained were studied and performed using Graphpad Prisms 8.0. Data shown as mean \pm standard deviation. One-way ANOVA tool was used to analyze the significant difference between groups and a value of $\mathrm{p}<0.05$ was considered statistically significant.

\section{Results}

The mean particle size, polydispersity index (PDI) and zeta potential of the quercetin formulation were measured and summarized in table 1. The mean particle size of the LCN formulations ranged from 210.0 to $268.7 \mathrm{~nm}$ with PDI less than 0.4 for all preparations. The zeta potentials for LCN formulations ranged from -14.6 to $-15.6 \mathrm{mV}$ whereas the sm-LCN ranged from 15.8 to 22.3 $\mathrm{mV}$. The encapsulation efficiency of quercetin loaded LCN was $99.4 \%$ which was slightly higher compared to the surface modified LCNs.

The TEM images (Figure 2) show that both LCN and sm-LCN were spherical in shape. The particle size of LCNs ranged from $100 \mathrm{~nm}$ to $300 \mathrm{~nm}$ which was consistent with the dynamic light scattering. 
Media only condition represented as negative control while LPS only condition represented as positive control. The significant difference at $\mathrm{p}<0.05$ of pro-inflammatory cytokines were indicated with the '*' sign above the bar when compared to negative control whereas the "\#” sign for positive control.

The BCi-NS1.1 cells were treated simultaneously with either $100 \mathrm{ng} / \mathrm{mL}$ of LPS alone or with quercetin loaded LCNs and free quercetin in DMSO solution to investigate their respective effects on the production of LPS induced pro-inflammatory markers in these cell lines. Quercetin loaded LCNs and sm-LCNs with the dose of $25 \mu \mathrm{M}$ showed a consistent trend of significantly (p $<0.05$ ) decreasing the concentration of pro-inflammatory cytokines namely IL-1 $\beta$, IL- 6 and IL-8 compared to the positive control group (Figure 4). It is also worth noting that quercetin LCNs as well as sm-LCNs remarkably downregulated the production of IL-6, IL-8 and IL-1 $\beta$ compared to free quercetin dissolved in DMSO, therefore, exhibiting superior anti-inflammatory activity.

\section{Discussion}

\subsection{Characterization of $L C N$ and sm-LCN}

The significant increase in particle size indicates the encapsulation of quercetin into the blank LCN. Drug encapsulation into the nanoparticles may be a result of chemical conjugation or physical entrapment [36]. Polydispersity index (PDI) represents the size distribution and uniformity of the nanoparticles formed [37]. PDI values greater than 0.3 indicates a broad size distribution. Ideally, a narrow size distribution identified with PDI values lesser than 0.3 is preferred [37]. The slightly higher polydispersity index of quercetin loaded LCNs may be attributed to the coexistence of cubic liquid crystalline nanoparticles with other types of vesicles in the dispersion as previously reported $[38,39]$. It was observed that the mean particle size for sm-LCN was significantly larger than LCN with regards to the amount of CHI used to modify the surface. When $0.1 \%$ CHI was used, the particle size observed was $236.1 \mathrm{~nm}$ whereas $0.2 \%$ CHI used has shown to increase the particle size to $268.7 \mathrm{~nm}$. The size increased in proportion to the quantity of $\mathrm{CHI}$ used proves that the increment was attributed to the surface coating of $\mathrm{CHI}$ on LCN [13]. 
The positive reading in zeta potential further indicates that the surface of LCN (negatively charged) have been successfully coated by the protonated amino group from CHI (13). Generally, zeta potential values above $\pm 25 \mathrm{mV}$ are required to form a stable dispersion between particles and reduce their aggregation [40]. P 407 aided in stabilising the LCN dispersion as demonstrated previously [41]. It is presumed that the hydrophobic part of the polypropylene oxide chain adheres to the surface of the cubic nanoparticles whereas the hydrophilic portion of the chain extends out to the surrounding environment to provide steric shielding, therefore, avoiding agglomeration [42]. The negatively charged zeta potential could be ascribed to the subtle amount of oleic acid released by monoolein resulting in anionic behavior of the dispersion caused by adsorption of hydroxyl ions on the surface of the LCNs [40, 42].

TEM images further confirmed the results from the Zetasizer, showing that the particles were spherical in shape and size less than $300 \mathrm{~nm}$, which is quite similar to other reported studies $[13,16,41,43]$. The dense black core surrounding the particle was due to the existence of CHI coating indicating that the surface of $\mathrm{LCN}$ has been modified.

Both LCN and sm-LCN have exhibited a high entrapment efficiency whereby $>97 \%$ of drug was successfully entrapped. This indicates that most of the drug has been encapsulated regardless the surface modification of the nanoparticle. However other studies have successfully encapsulated their drug as high as $99 \%$ in $\operatorname{LCN}[13,16,40]$. Nevertheless, the entrapment efficiency is still considered high when compared to other nanocarriers such as poly lactide-coglycolic acid (PLGA) polymer-based nanoparticles where Anwar et al reported that only $86 \%$ of quercetin was encapsulated [44].

\subsection{In vitro release study}

The result has shown that quercetin drug was able to release in a sustained release manner when incorporated into the LCN and by modifying the surface with $\mathrm{CHI}$ further prolonged the drug release. This sustained release of quercetin could be attributed to the structure of the LCN. The higher proportion of lipids in the LCN not only allows hydrophobic drug to bind strongly to its inner structure, but also provides a larger surface area for the drugs to be incorporated. As a result, high entrapment efficiency of the drug can be achieved. Yoo et al., also showed that the release of the drug from LCN has sustained as long as 15 days [16]. 


\subsection{In vitro evaluation anti-inflammatory activity of quercetin loaded LCN and sm-LCN on immortalised human bronchial epithelial cell line (BCi-NS1.1)}

Evaluation of in vitro anti-inflammatory activity of quercetin loaded $L C N$ and sm-LCN in comparison to free quercetin in DMSO solution

In the present study, quercetin loaded LCNs effectively decreased the concentration of proinflammatory cytokines namely IL-1 $\beta$, IL- 6 and IL-8. These effects exhibited by quercetin LCN was in consistent with previous studies evaluating the anti-inflammatory activity of quercetin on different cell lines $[9,11,45-47]$. Zhang et al., proved that quercetin remarkably suppressed the secretion of IL-6 and IL-1 $\beta$ in LPS-stimulated human peripheral blood mononuclear cells (PBMCs) [46]. Another research demonstrated that the treatment of quercetin notably reduced the gene expression and release of IL-8, IL-6 as well as IL-1 $\beta$ in human mast cells (HMC-1) induced by phorbol 12-myristate 13-acetate and calcium ionophore A23187 [9]. Although the underlying mechanism was not investigated in this present study, it is well-established from previous studies that quercetin attenuates the activation of NF- $\mathrm{kB}$ hence, downregulating the production of pro-inflammatory cytokines $[9,45,46]$. NF- $\mathrm{KB}$ signaling pathway plays a crucial role in the pathogenesis of asthma as it modulates the gene expression of pro-inflammatory mediators including chemokines, cytokines and cell adhesion molecules that lead to the infiltration of immune cells such as neutrophils and eosinophils in the airway $[48,49]$.

Encapsulation of quercetin into liquid crystalline nanoparticles was successful in further improving its anti-inflammatory activity. The enhanced anti-inflammatory effects of quercetin LCNs could be primarily attributed to the characteristics of the LCN itself, whereby it is bioadhesive in nature and improves membrane permeation leading to an increased interaction with the bronchial epithelial cells $[40,50]$. The lipid bilayer of LCNs is akin to the epithelial cell membrane hence could be transported easily across the membrane resulting in greater drug absorption [50, 51]. Furthermore, findings from numerous studies also elucidated that LCNs promote greater cellular uptake and internalisation into cells via endocytosis enabling them to be diffused throughout the cytoplasm [16, 40,51,52]. On the other hand, quercetin itself could have only remained in the cell membrane due to its high lipophilicity allowing it to be easily incorporated into the cell membrane. 
The findings observed in our study has demonstrated that quercetin loaded sm-LCN with the dose of $25 \mu \mathrm{M}$ significantly $(\mathrm{p}<0.05)$ decreased the pro-inflammatory markers when compared to the free quercetin drug in DMSO. This indicates that sm-LCN is able to enhance the anti-inflammatory effect of quercetin when entrapped in the nanocarrier.

There is also a noticeable reduction in the expression of all the pro-inflammatory cytokines which was observed on the blank LCN \& sm-LCN. This suggests that the empty vehicle itself can slightly reduce the inflammation. We suspect that one of the components in the LCN formulation might contributed and it was monoolein. However, only one study has confirmed this and Ali et al reported that monoolein possess anti-inflammatory property that inhibits the production of pro-inflammatory markers in LPS stimulated bone marrow-derived dendritic cell (BMDC) via NF- $\mathrm{BB}$ and MAPK pathways [53].

Evaluation of in vitro anti-inflammatory activity of quercetin loaded $L C N$ and $s m-L C N$ in comparison to fluticasone

Fluticasone, an FDA approved drug to treat asthma was used as a standard to further establish the anti-inflammatory activity of quercetin LCNs. Quercetin LCNs were comparable to fluticasone in terms of reducing the levels of IL-1 $\beta$ and IL-6 however, fluticasone was much more effective in decreasing the production of IL-8. In this existing study, the BCi-NS1.1 cell lines were only treated with quercetin LCNs at the dose of $25 \mu \mathrm{M}$. Quercetin was able to reduce the levels of pro-inflammatory cytokines in a dose-dependent manner up to a $100 \mu \mathrm{M}$ as described in the literature [46]. Therefore, the finding suggests the potential of quercetin LCNs to decrease the levels pro-inflammatory cytokines to a greater extent comparable to fluticasone at a higher dose.

\subsection{Molecular mechanics simulations}

The molecular simulations performed in this research focused on two aspects: a) encapsulation or incorporation of quercetin within a monoolein matrix, and b) surface modification of the monoolein matrix with chitosan. The geometrical iterations representing the above bimolecular complexes are shown in Figure 5 while the related energetic molecular attributes are depicted in Tables 2 and 3, respectively. In case of MO-Quer; the formation of molecular complex was accompanied by a total energy stabilization of $15.894 \mathrm{kcal} / \mathrm{mol}$. Interestingly, the total energy of 
the MO-Quer complex was less than the individual constituent molecules. The incorporation of Quer in MO was evident from the fact that all bonding (bond, angle, and dihedral) and nonbonding (van der waals forces and H-bonding) energy terms contributed to the geometrical stabilization of the complex. The major stabilization was provided by the van der Waals function which brought the molecules in close vicinity and hence led to the formation of -OH...HObonds between the molecules (Table 2, Figure 5). For the MO-Cht molecular complex; an energy stabilization of $10.336 \mathrm{kcal} / \mathrm{mol}$ was observed. In line with MO-Quer, the total energy of the MOCht complex was less than the individual molecular components. The energy stabilization in this case was mainly due to non-bonding interactions (van der Waals forces and electrostatic interactions) while the bonding interactions (bond and dihedral energies) destabilized the complex. Such destabilization is due to torsional strain experienced by the molecules while interacting within the van der Waals space. Additionally, both inter- and intra-molecular $\mathrm{H}$ bonding was observed in the MO-Cht complex further confirming its favourable formation and conformation. The above discussion justifies the selection and successful incorporation of quercetin in and chitosan surface modification of the monoolein nanosystem.

\section{Conclusions}

Quercetin was successfully encapsulated into liquid crystalline nanoparticles revealing their sustained release behaviour. Moreover, quercetin LCN and sm-LCN were effective in reducing the levels of pro-inflammatory mediators such as IL-1 $\beta$, IL-6 and IL- 8 whereby encapsulating quercetin into LCN further enhanced its anti-inflammatory activity. This demonstrates that the quercetin LCN could be employed as a potential novel drug delivery for the treatment of asthma. This mode will overcome the issues associated with quercetin such as the low solubility, poor bioavailability and short half-life. The findings of this present study advocate the translation into other associated pulmonary inflammatory diseases such as chronic obstructive pulmonary disorder (COPD) as well as lung cancer which will help in providing a new direction to the pulmonary clinics.

\section{References}

[1]. Tanaka T., Takahashi R. Flavonoids and asthma. Nutrients. 5 (2013) 2128-43. 
[2]. Asthma, Facts about asthma, World Health Organization. https://www.who.int/newsroom/fact-sheets/detail/asthma, 2017 (accessed 6 September 2019).

[3]. Pandya D., Puttanna A., Balagopal V. Suppl 1: M2: Systemic Effects of Inhaled Corticosteroids: An Overview. The open respiratory medicine journal. 8 (2014) 59.

[4]. Hansbro P.M., Kim R.Y., Starkey M.R., Donovan C., Dua K., Mayall J.R., et al. Mechanisms and treatments for severe, steroid-resistant allergic airway disease and asthma. Immunological reviews. 278 (2017) 41-62.

[5]. Dua K., Hansbro N.G., Hansbro P.M. Steroid resistance and concomitant respiratory infections: A challenging battle in pulmonary clinic. EXCLI journal. 16 (2017) 981.

[6]. Wang W., Sun C., Mao L., Ma P., Liu F., Yang J., et al. The biological activities, chemical stability, metabolism and delivery systems of quercetin: A review. Trends in Food Science \& Technology. 56 (2016) 21-38.

[7]. D'Andrea G. Quercetin: a flavonol with multifaceted therapeutic applications? Fitoterapia. 106 (2015) 256-71.

[8]. Cruz E., Reuter S., Martin H., Dehzad N., Muzitano M., Costa S., et al. Kalanchoe pinnata inhibits mast cell activation and prevents allergic airway disease. Phytomedicine. 19 (2012) 11521.

[9]. Park H.-H., Lee S., Son H.-Y., Park S.-B., Kim M.-S., Choi E.-J., et al. Flavonoids inhibit histamine release and expression of proinflammatory cytokines in mast cells. Archives of pharmacal research. 31 (2008) 1303.

[10]. Oliveira T.T., Campos K.M., Cerqueira-Lima A.T., Carneiro T.C.B., da Silva Velozo E., Melo I.C.A.R., et al. Potential therapeutic effect of Allium cepa L. and quercetin in a murine model of Blomia tropicalis induced asthma. DARU Journal of Pharmaceutical Sciences. 23 (2015) 18. 
[11]. Takashima K., Matsushima M., Hashimoto K., Nose H., Sato M., Hashimoto N., et al. Protective effects of intratracheally administered quercetin on lipopolysaccharide-induced acute lung injury. Respiratory research. 15 (2014) 150.

[12]. Sharma S., Ali A., Ali J., Sahni J.K., Baboota S. Rutin: therapeutic potential and recent advances in drug delivery. Expert opinion on investigational drugs. 22 (2013) 1063-79.

[13]. Madheswaran T., Baskaran R., Sundaramoorthy P., Yoo B.K. Enhanced skin permeation of $5 \alpha$-reductase inhibitors entrapped into surface-modified liquid crystalline nanoparticles. Archives of pharmacal research. 38 (2015) 534-42.

[14]. Mulet X., Boyd B.J., Drummond C.J. Advances in drug delivery and medical imaging using colloidal lyotropic liquid crystalline dispersions. Journal of colloid and interface science. 393 (2013) 1-20.

[15]. Madheswaran T., Baskaran R., Yong C.S., Yoo B.K. Enhanced topical delivery of finasteride using glyceryl monooleate-based liquid crystalline nanoparticles stabilized by cremophor surfactants. AAPS PharmSciTech. 15 (2014) 44-51.

[16]. Baskaran R., Madheswaran T., Sundaramoorthy P., Kim H.M., Yoo B.K. Entrapment of curcumin into monoolein-based liquid crystalline nanoparticle dispersion for enhancement of stability and anticancer activity. International journal of nanomedicine. 9 (2014) 3119.

[17]. Dua K., Shukla S.D., de Jesus Andreoli Pinto T., Hansbro P.M. Nanotechnology: Advancing the translational respiratory research. Interventional Medicine and Applied Science. 9 (2017) 39-41.

[18]. Choi S.W., Kim W.S., Kim J.H. Surface-functionalized nanoparticles for controlled drug delivery. Methods in molecular biology (Clifton, NJ). $303 \quad$ (2005) 121-31. https://doi.org/10.1385/1-59259-901-x:121.

[19]. Al-Nemrawi N.K., Okour A.R., Dave R.H. Surface modification of PLGA nanoparticles using chitosan: Effect of molecular weight, concentration, and degree of deacetylation. Advances in Polymer Technology. 37 (2018) 3066-75. 
[20]. Dua K., Malyla V., Singhvi G., Wadhwa R., Krishna R.V., Shukla S.D., et al. Increasing complexity and interactions of oxidative stress in chronic respiratory diseases: An emerging need for novel drug delivery systems. Chemico-biological interactions. (2018).

[21]. Dua K., Madan J.R., Chellappan D.K., Gupta G. Nanotechnology in drug delivery gaining new perspectives in respiratory diseases. Panminerva medica. 60 (2018) 135.

[22]. Dua K., Rapalli V.K., Shukla S.D., Singhvi G., Shastri M.D., Chellappan D.K., et al. Multidrug resistant Mycobacterium tuberculosis \& oxidative stress complexity: Emerging need for novel drug delivery approaches. Biomedicine \& Pharmacotherapy. 107 (2018) 1218-29.

[23]. Mehta M., Sharma N., Vyas M., Khurana N., Maurya P.K., Singh H., et al. Interactions with the macrophages: An emerging targeted approach using novel drug delivery systems in respiratory diseases. Chemico-biological interactions. (2019).

[24]. Ng Z.Y., Wong J.-Y., Panneerselvam J., Madheswaran T., Kumar P., Pillay V., et al. Assessing the potential of liposomes loaded with curcumin as a therapeutic intervention in asthma. Colloids and Surfaces B: Biointerfaces. 172 (2018) 51-9.

[25]. Das P., Kumar K., Nambiraj A., Rajan R., Awasthi R., Dua K., et al. Potential therapeutic activity of Phlogacanthus thyrsiformis Hardow (Mabb) flower extract and its biofabricated silver nanoparticles against chemically induced urolithiasis in male Wistar rats. International journal of biological macromolecules. 103 (2017) 621-9.

[26]. Madan J.R., Kamate V.J., Awasthi R., Dua K. Formulation, Characterization and In-vitro Evaluation of Fast Dissolv ing Tablets Containing Gliclazide Hydrotropic Solid Dispersions. Recent patents on drug delivery \& formulation. $11 \quad$ (2017) 147-54. https://doi.org/10.2174/1872211311666170427100213.

[27]. Chellappan D.K., Yee N.J., Kaur Ambar Jeet Singh B.J., Panneerselvam J., Madheswaran T., Chellian J., et al. Formulation and characterization of glibenclamide and quercetin-loaded chitosan nanogels targeting skin permeation. Therapeutic delivery. 10 (2019) 281-93.

[28]. Tambuwala M.M., Khan M.N., Thompson P., McCarron P.A. Albumin nano-encapsulation of caffeic acid phenethyl ester and piceatannol potentiated its ability to modulate HIF and NF-kB 
pathways and improves therapeutic outcome in experimental colitis. Drug delivery and translational research. 9 (2019) 14-24.

[29]. Abdelkader D.H., Tambuwala M.M., Mitchell C.A., Osman M.A., El-Gizawy S.A., Faheem A.M., et al. Enhanced cutaneous wound healing in rats following topical delivery of insulin-loaded nanoparticles embedded in poly (vinyl alcohol)-borate hydrogels. Drug delivery and translational research. 8 (2018) 1053-65.

[30]. Khan M.N., Haggag Y.A., Lane M.E., McCarron P.A., Tambuwala M.M. Polymeric nanoencapsulation of curcumin enhances its anti-cancer activity in breast (MDA-MB231) and lung (A549) cancer cells through reduction in expression of HIF-1 $\alpha$ and nuclear p65 (REL A). Current drug delivery. 15 (2018) 286-95.

[31]. Das P., Kumar K., Nambiraj A., Awasthi R., Dua K., Malipeddi H. Antibacterial and In Vitro Growth Inhibition Study of Struvite Urinary Stones Using Oxalis corniculata Linn. Leaf Extract and its Biofabricated Silver Nanoparticles. Recent patents on drug delivery \& formulation. 12 (2018) 170-8.

[32]. Malipeddi V.R., Awasthi R., Dua K. Formulation and evaluation of controlled release ethylcellulose and polyethylene glycol microspheres containing metoprolol tartrate. Interventional Medicine and Applied Science. 8 (2016) 60-7.

[33]. Hsu A.C., Dua K., Starkey M.R., Haw T.-J., Nair P.M., Nichol K., et al. MicroRNA-125a and-b inhibit A20 and MAVS to promote inflammation and impair antiviral response in COPD. JCI insight. 2 (2017).

[34]. Baines K.J., Hsu A.C., Tooze M., Gunawardhana L.P., Gibson P.G., Wark P.A. Novel immune genes associated with excessive inflammatory and antiviral responses to rhinovirus in COPD. Respiratory research. 14 (2013) 15.

[35]. Kumar P., Choonara Y.E., Pillay V. In silico analytico-mathematical interpretation of biopolymeric assemblies: Quantification of energy surfaces and molecular attributes via atomistic simulations. Bioengineering \& translational medicine. 3 (2018) 222-31. 
[36]. He X., Li Q., Liu X., Wu G., Zhai G. Curcumin-loaded lipid cubic liquid crystalline nanoparticles: preparation, optimization, physicochemical properties and oral absorption. Journal of nanoscience and nanotechnology. 15 (2015) 5559-65.

[37]. Danaei M., Dehghankhold M., Ataei S., Hasanzadeh Davarani F., Javanmard R., Dokhani A., et al. Impact of particle size and polydispersity index on the clinical applications of lipidic nanocarrier systems. Pharmaceutics. 10 (2018) 57.

[38]. Nasr M., Ghorab M.K., Abdelazem A. In vitro and in vivo evaluation of cubosomes containing 5-fluorouracil for liver targeting. Acta pharmaceutica sinica B. 5 (2015) 79-88.

[39]. Nanjwade B.K., Hundekar Y.R., Kamble M., Srichana T. Development of cuboidal nanomedicine by nanotechnology. Austin J Nanomed Nanotechnol. 2 (2014) 1023.

[40]. Freag M.S., Elnaggar Y.S., Abdelmonsif D.A., Abdallah O.Y. Stealth, biocompatible monoolein-based lyotropic liquid crystalline nanoparticles for enhanced aloe-emodin delivery to breast cancer cells: in vitro and in vivo studies. International journal of nanomedicine. 11 (2016) 4799.

[41]. Madheswaran T., Baskaran R., Thapa R.K., Rhyu J.Y., Choi H.Y., Kim J.O., et al. Design and in vitro evaluation of finasteride-loaded liquid crystalline nanoparticles for topical delivery. AAPS PharmSciTech. 14 (2013) 45-52.

[42]. Verma P., Ahuja M. Cubic liquid crystalline nanoparticles: optimization and evaluation for ocular delivery of tropicamide. Drug delivery. 23 (2016) 3043-54.

[43]. Thapa R.K., Baskaran R., Madheswaran T., Kim J.O., Yong C.S., Yoo B.K. Preparation, characterization, and release study of tacrolimus-loaded liquid crystalline nanoparticles. Journal of Dispersion Science and Technology. 34 (2013) 72-7.

[44]. Anwer M.K., Al-Mansoor M.A., Jamil S., Al-Shdefat R., Ansari M.N., Shakeel F. Development and evaluation of PLGA polymer based nanoparticles of quercetin. Int J Biol Macromol. 92 (2016) 213-9. https://doi.org/10.1016/j.ijbiomac.2016.07.002. 
[45]. Min Y.D., Choi C.H., Bark H., Son H.Y., Park H.H., Lee S., et al. Quercetin inhibits expression of inflammatory cytokines through attenuation of NF-kappaB and p38 MAPK in HMC-1 human mast cell line. Inflammation research : official journal of the European Histamine Research Society [et al]. 56 (2007) 210-5. https://doi.org/10.1007/s00011-007-6172-9.

[46]. Zhang M., Lin J.M., Li X.S., Li J. Quercetin ameliorates LPS-induced inflammation in human peripheral blood mononuclear cells by inhibition of the TLR2-NF-kappaB pathway. Genetics and molecular research : GMR. 15 (2016). https://doi.org/10.4238/gmr.15028297.

[47]. Weng Z., Zhang B., Asadi S., Sismanopoulos N., Butcher A., Fu X., et al. Quercetin is more effective than cromolyn in blocking human mast cell cytokine release and inhibits contact dermatitis and photosensitivity in humans. PloS one. 7 (2012) e33805. https://doi.org/10.1371/journal.pone.0033805.

[48]. Schuliga M. NF-kappaB Signaling in Chronic Inflammatory Airway Disease. Biomolecules. 5 (2015) 1266-83. https://doi.org/10.3390/biom5031266.

[49]. Starkey M., Hanish I., Dua K., Nair P., Haw T., Hsu A., et al. 175: Interleukin-13 predisposes mice to more severe influenza infection by suppressing interferon responses and activating microRNA-21/PI3K. Cytokine. 70 (2014) 70.

[50]. Jin X., Zhang Z.-h., Li S.-1., Sun E., Tan X.-b., Song J., et al. A nanostructured liquid crystalline formulation of 20 (S)-protopanaxadiol with improved oral absorption. Fitoterapia. 84 (2013) 64-71.

[51]. Luo Q., Lin T., Zhang C.Y., Zhu T., Wang L., Ji Z., et al. A novel glyceryl monooleinbearing cubosomes for gambogenic acid: preparation, cytotoxicity and intracellular uptake. International journal of pharmaceutics. 493 (2015) 30-9.

[52]. Abdel-Bar H.M., el Basset Sanad R.A. Endocytic pathways of optimized resveratrol cubosomes capturing into human hepatoma cells. Biomedicine \& Pharmacotherapy. 93 (2017) $561-9$.

[53]. Ali I., Manzoor Z., Koo J.-E., Moon S.-R., Byeon S.-H., Yoo E.-S., et al. Monoolein, isolated from Ishige sinicola, inhibits lipopolysaccharide-induced inflammatory response by 


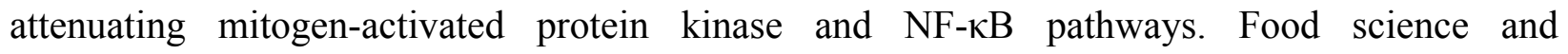
biotechnology. 26 (2017) 507-11. 


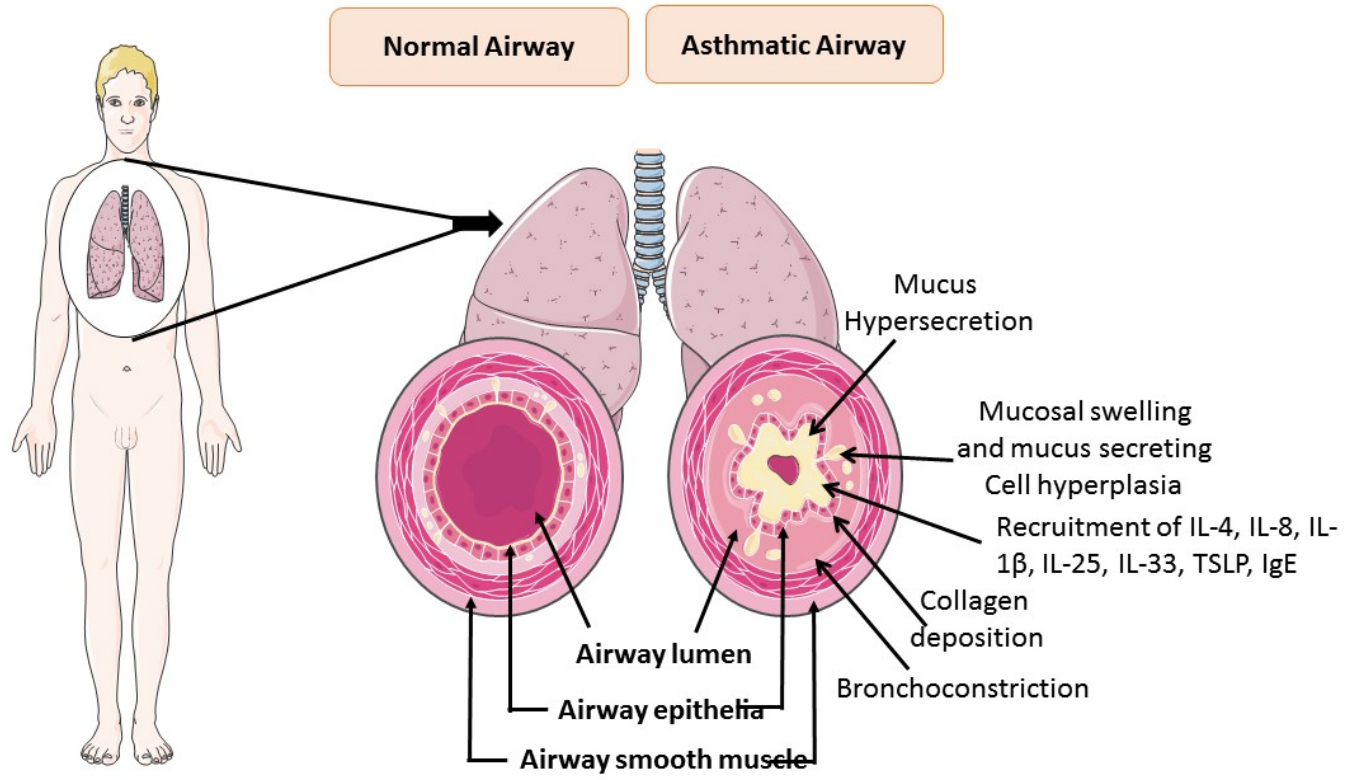

Figure 1: Pathology of Asthma<smiles>O=c1c(O)c(-c2ccc(O)c(O)c2)oc2cc(O)cc(O)c12</smiles>

Figure. 2. Chemical structure of Quercetin 


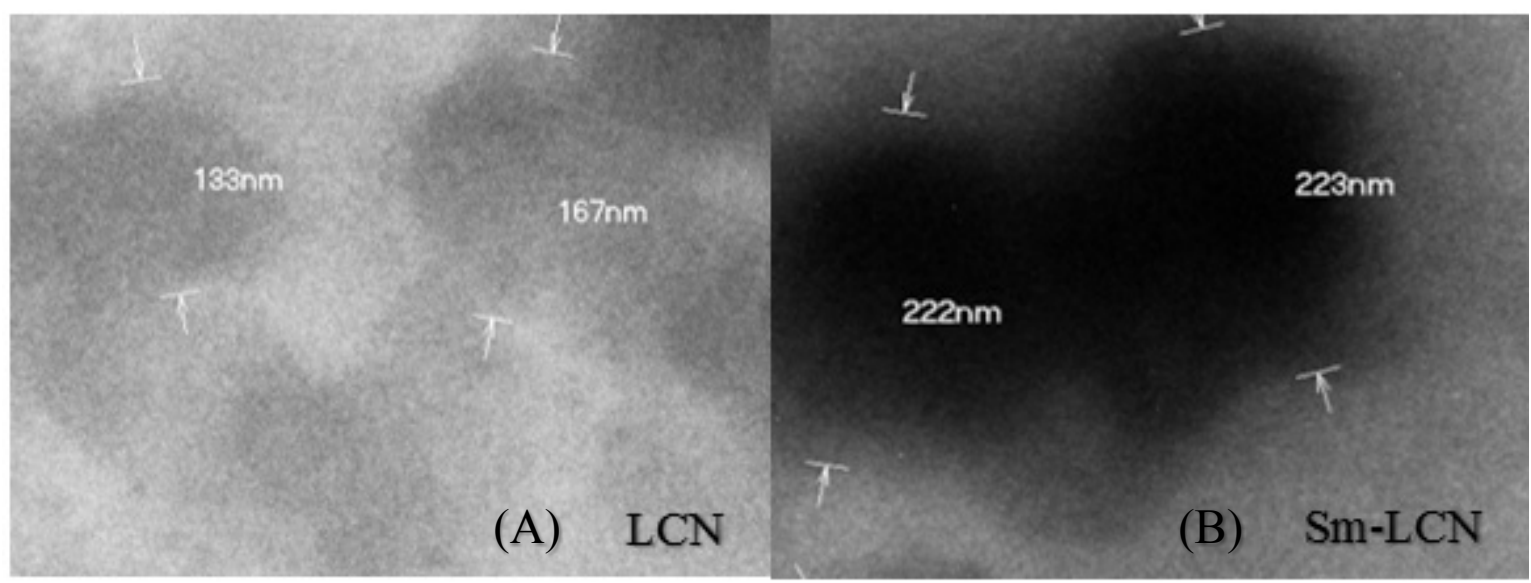

Figure 3. TEM images of LCN and sm-LCN (bar $500 \mathrm{~nm}$ ). (A) LCN liquid crystalline nanoparticle and (B) sm-LCN Surface-modified liquid crystalline nanoparticle.
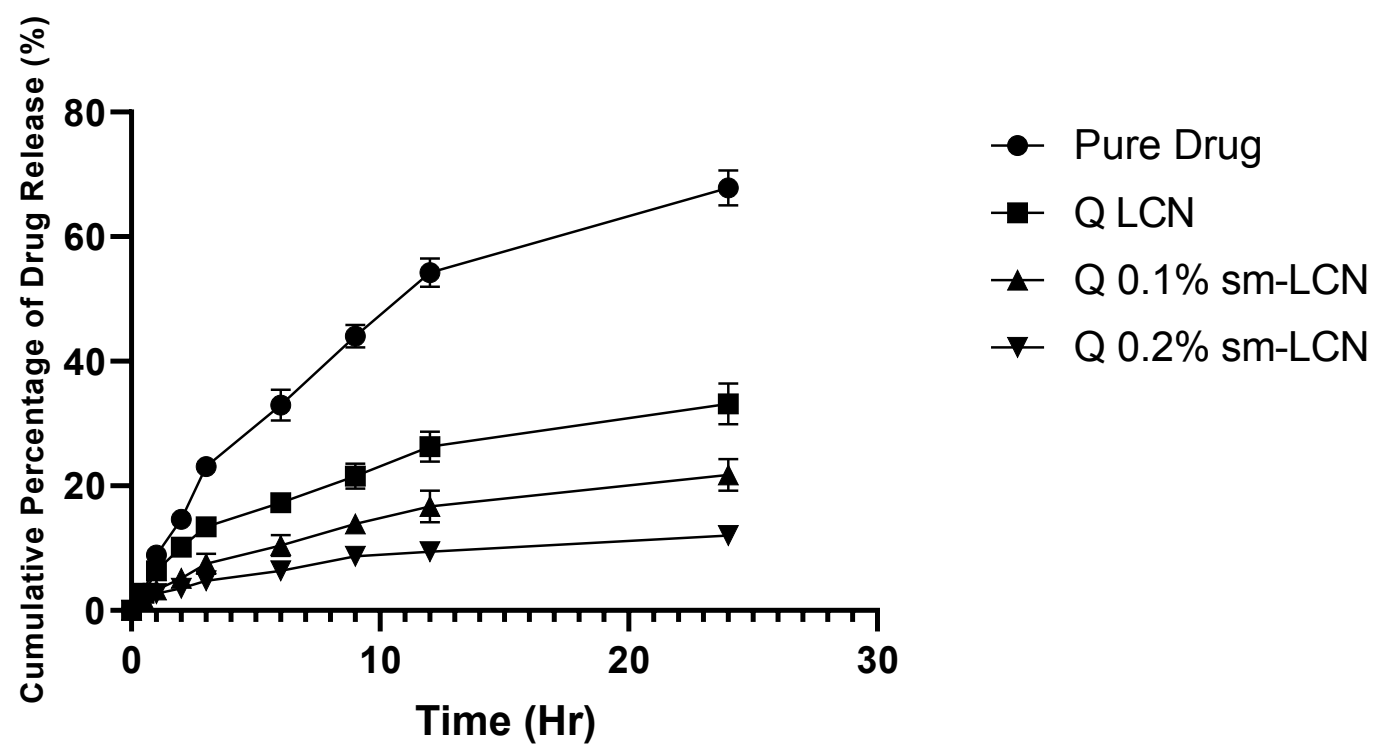

Figure 4. Cumulative Percentage release of Quercetin Vs. Time. The release profile of quercetin LCN over 24 hours. Release profile of quercetin from LCN exhibits a prolonged manner over the longer duration of time. 

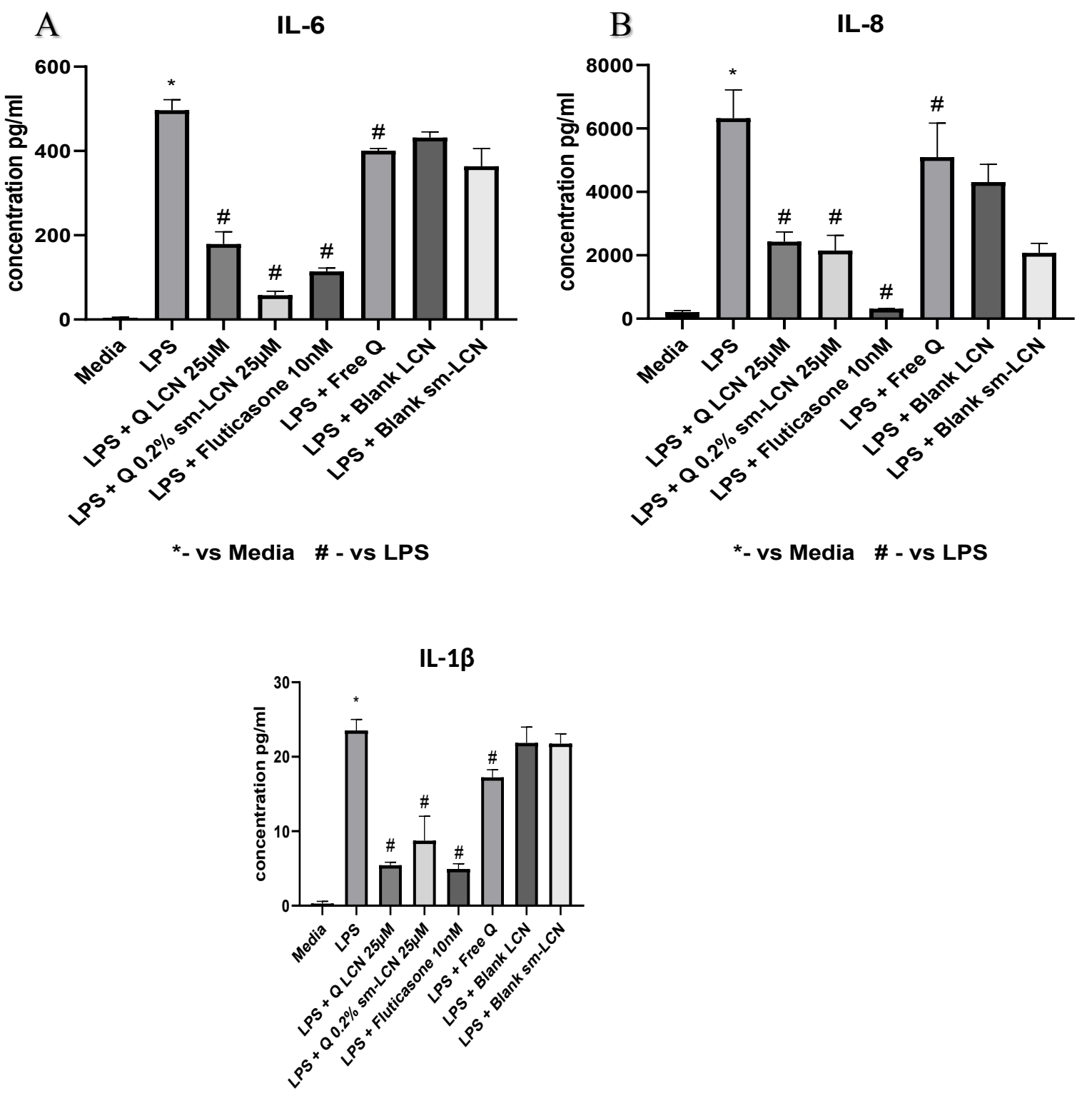

*-vs Media \#-vs LPS

Figure 5A, 5B and 5C. Effects of quercetin LCN treatment on BCi-NS1.1 cell line. Cells were treated with Q LCN, Q $0.2 \%$ sm-LCN at 25nM, fluticasone in DMSO at 10nM, Free Q in DMSO, Blank LCN and sm-LCN for $24 \mathrm{~h}(\mathrm{n}=3)$ 


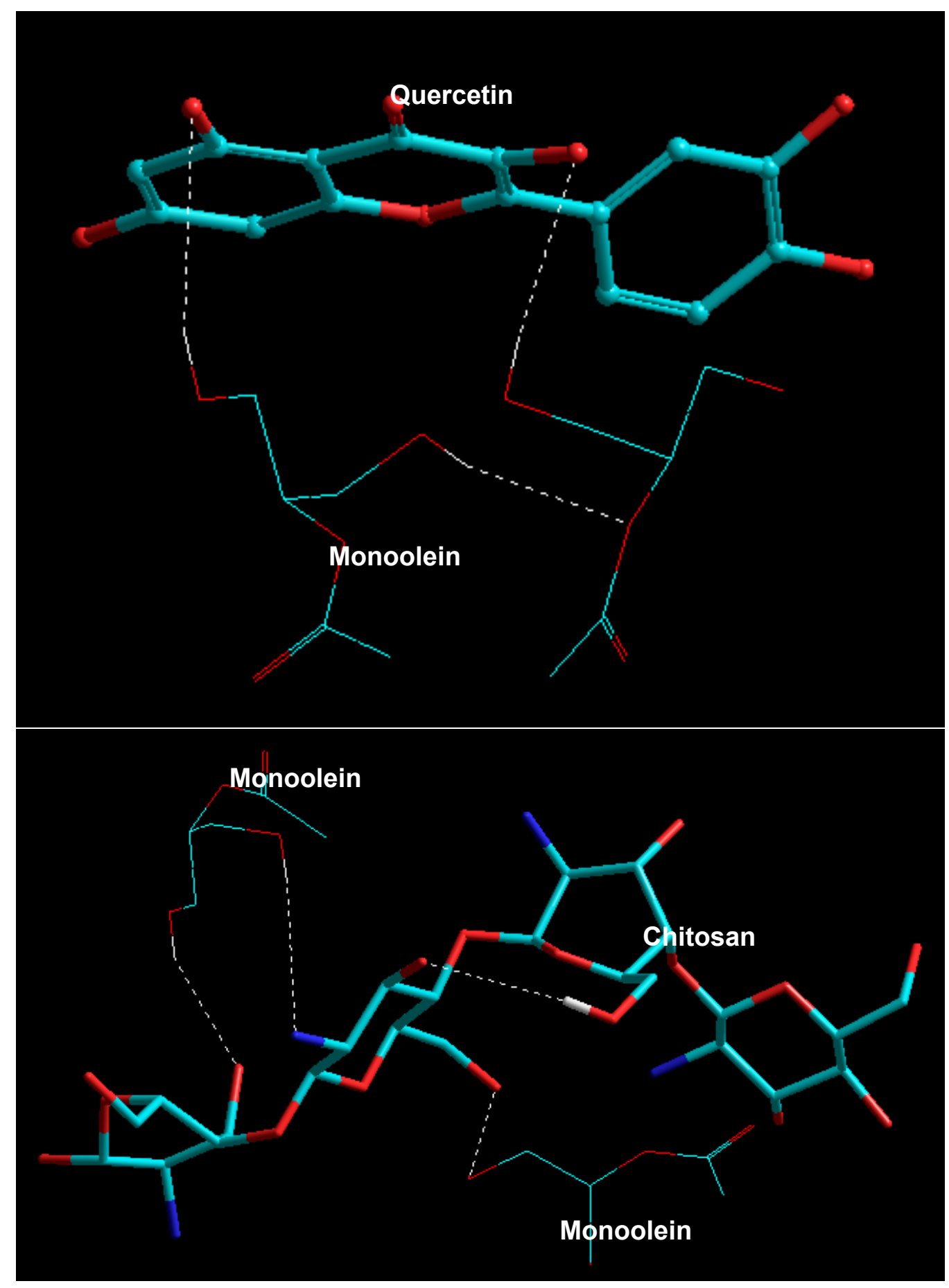

Figure 6: Representation of the geometrical preferences of the two monoolein functional moieties (stick rendering) in complexation with a) quercetin molecule (ball-and-tube rendering); and $b$ ) chitosan chain (tube rendering) after molecular mechanics simulations [Colour code for elements: $\mathrm{C}=$ cyan; $\mathrm{H}=$ white; $\mathrm{O}=$ red]. Hydrogen atoms not shown for clarity. 
<smiles>O=c1c(O)c(-c2ccc(O)c(O)c2)oc2cc(O)cc(O)c12</smiles>

Figure. 1. Chemical structure of Quercetin

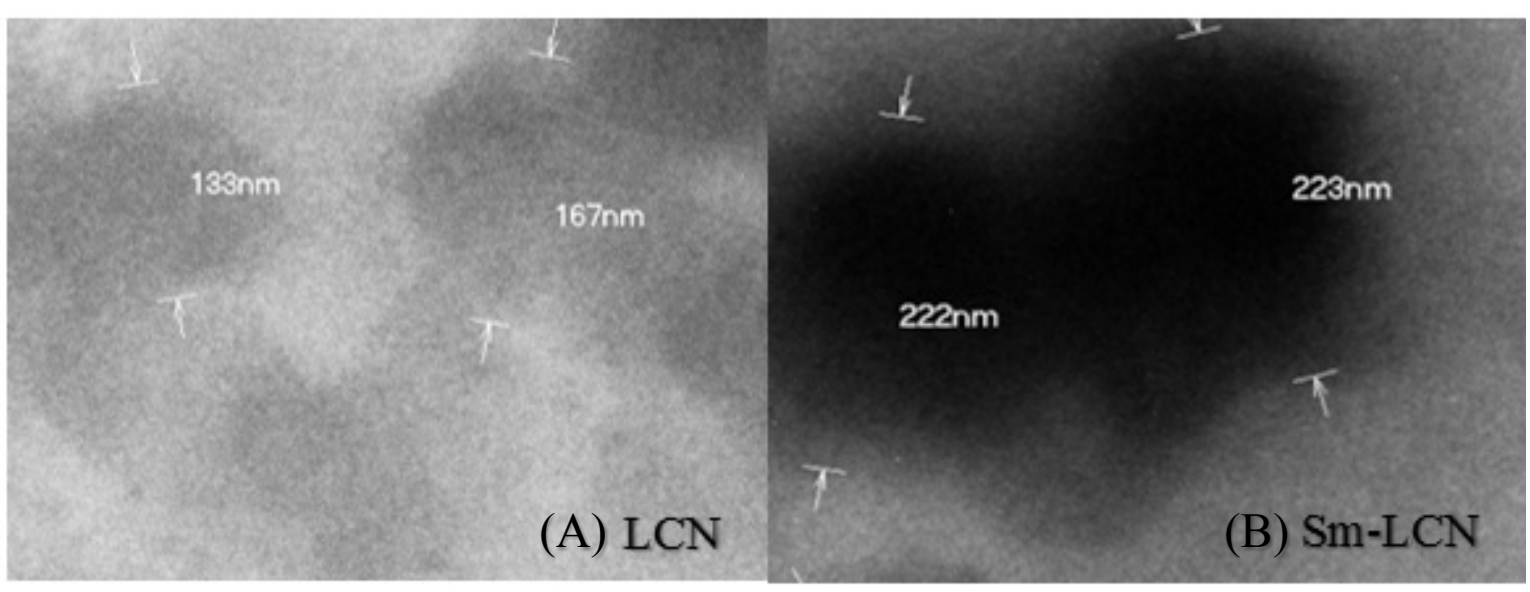

Figure 2. TEM images of LCN and sm-LCN (bar $500 \mathrm{~nm}$ ). (A) LCN liquid crystalline nanoparticle and (B) sm-LCN Surface-modified liquid crystalline nanoparticle.
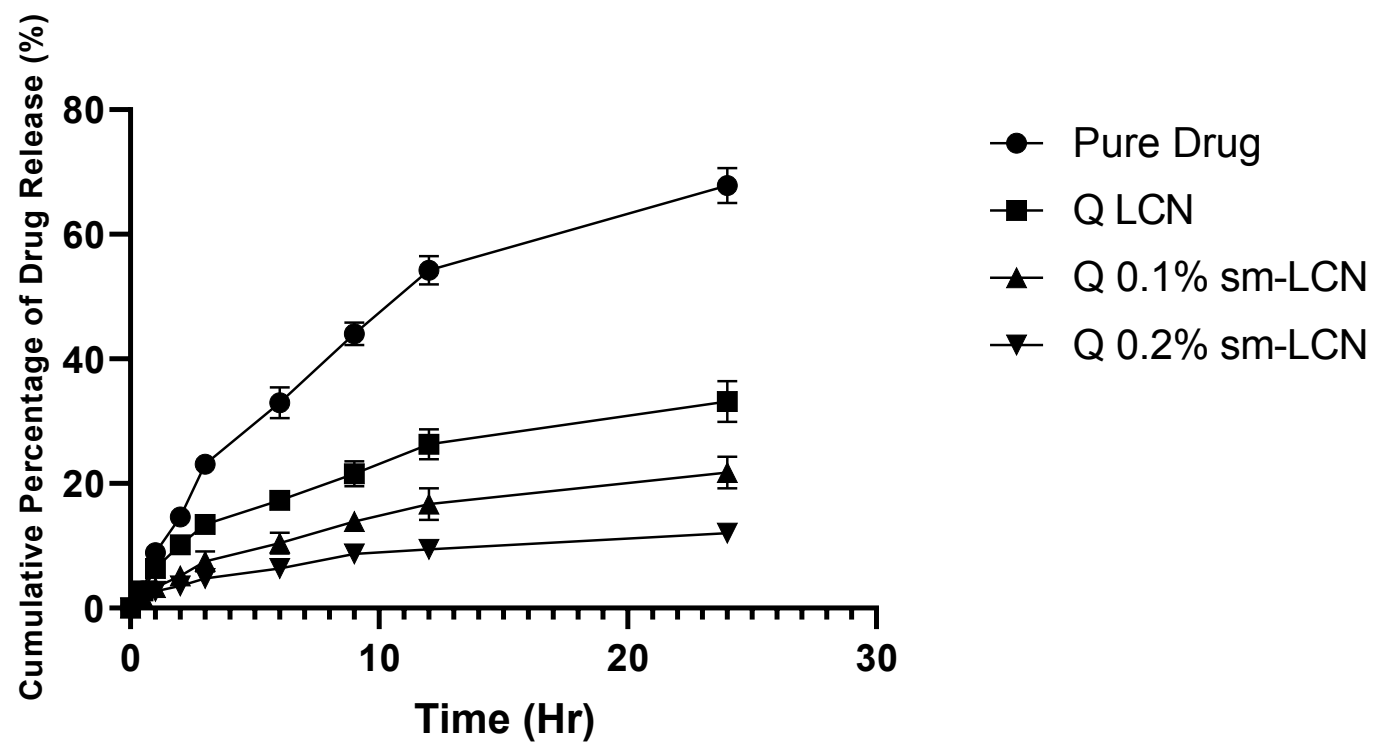

Figure 3. Cumulative Percentage release of Quercetin Vs. Time 
Figure 3 The release profile of quercetin LCN over 24 hours. Release profile of quercetin from LCN exhibits a prolonged manner over the longer duration of time.
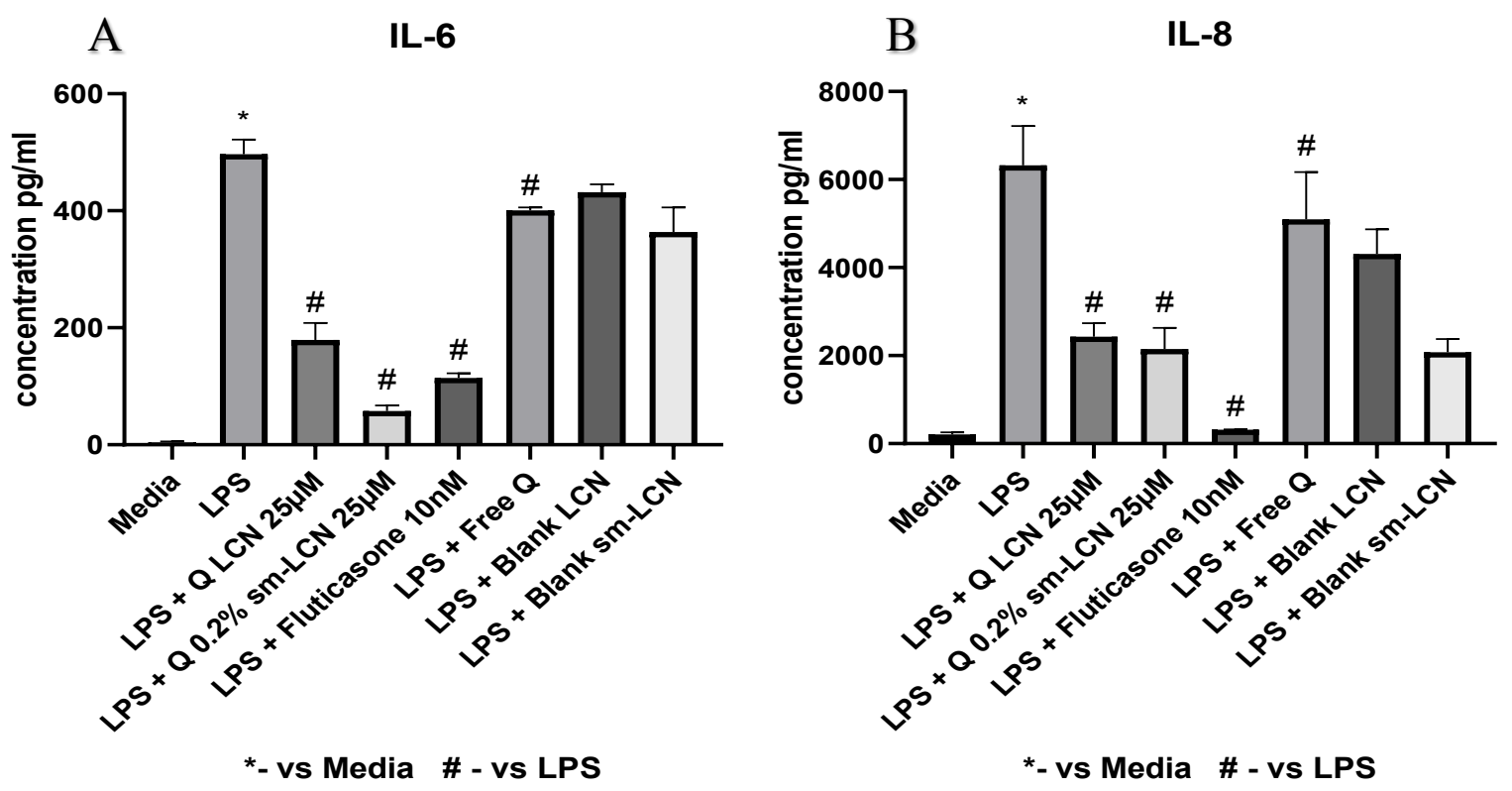

$\mathrm{C}$

IL-1b

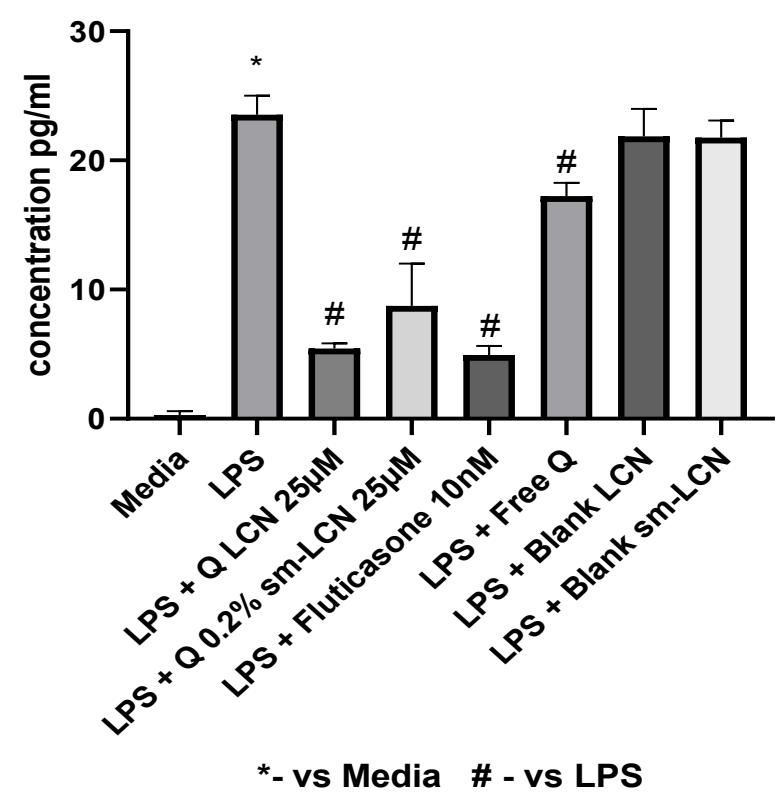

Figure 4A, 4B and 4C. Effects of quercetin LCN treatment on BCi-NS1.1 cell line. Cells were treated with Q LCN, Q $0.2 \%$ sm-LCN at $25 \mathrm{nM}$, fluticasone in DMSO at $10 \mathrm{nM}$, Free Q in DMSO, Blank LCN and sm-LCN for $24 \mathrm{~h}(\mathrm{n}=3)$ 


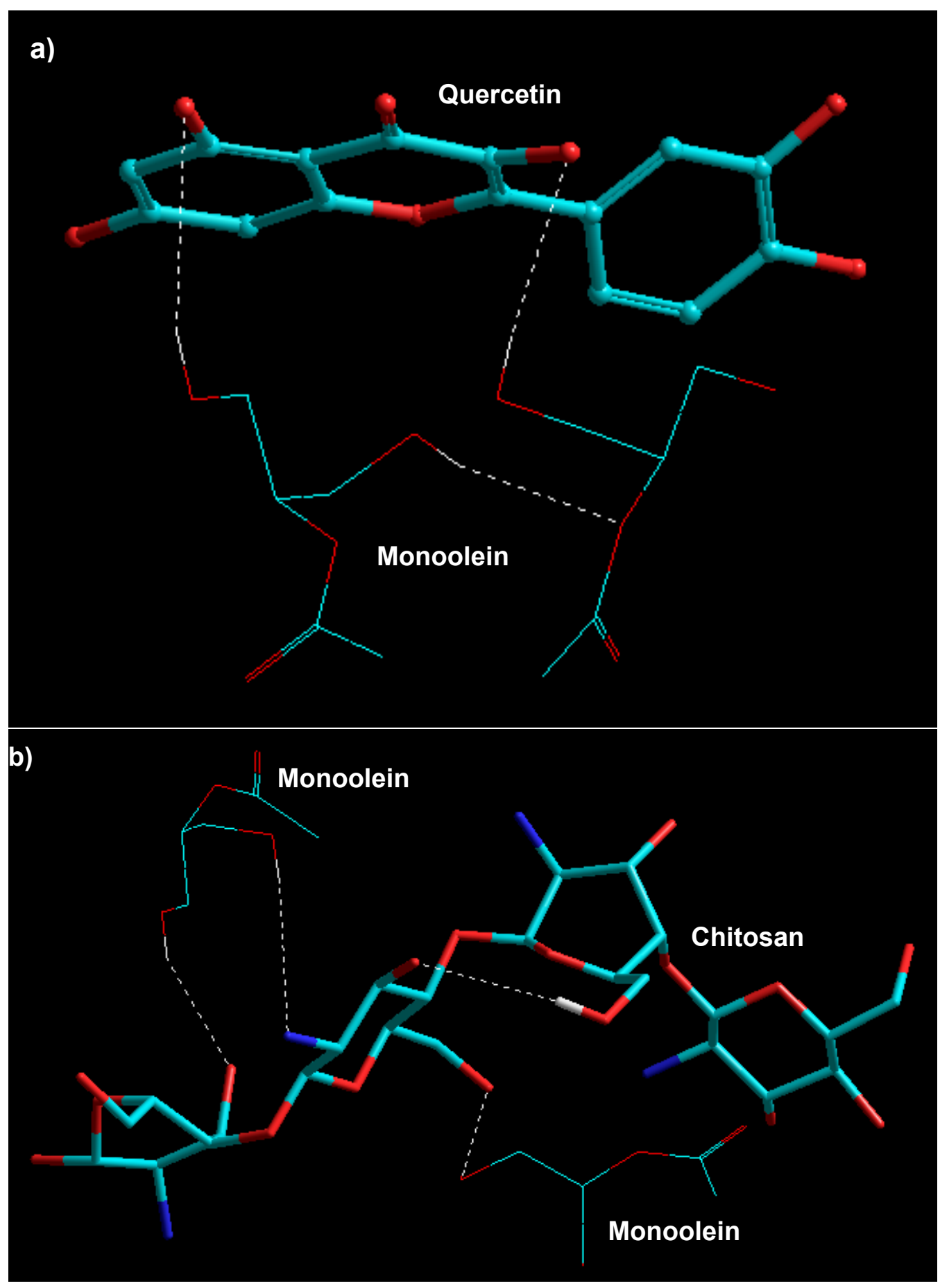

Figure 5: Representation of the geometrical preferences of the two monoolein functional moieties (stick rendering) in complexation with a) quercetin molecule (ball-and-tube rendering); and b) chitosan chain (tube rendering) after molecular mechanics simulations [Colour code for elements: $\mathrm{C}=$ cyan; $\mathrm{H}=$ white; $\mathrm{O}=$ red]. Hydrogen atoms not shown for clarity. 
Table 1. Characterization parameter of the nanoformulations.

\begin{tabular}{|l|l|l|l|l|}
\hline Formulations & $\begin{array}{l}\text { Mean particle } \\
\text { size (nm) }\end{array}$ & $\begin{array}{l}\text { Polydispersity } \\
\text { index (PDI) }\end{array}$ & $\begin{array}{l}\text { Zeta } \\
\text { potential } \\
(\mathbf{m V})\end{array}$ & $\begin{array}{l}\text { Encapsulation } \\
\text { efficiency (EE) } \\
\mathbf{( \% )}\end{array}$ \\
\hline Blank LCN & $210.0 \pm 2.77$ & $0.162 \pm 0.02$ & $-14.6 \pm 0.25$ & - \\
\hline Q LCN & $223.9 \pm 1.83$ & $0.339 \pm 0.008$ & $-15.6 \pm 0.17$ & $99.4 \pm 0.37$ \\
\hline $0.1 \%$ Q sm-LCN & $236.1 \pm 2.12$ & $0.384 \pm 0.03$ & $15.8 \pm 0.29$ & $97.6 \pm 0.30$ \\
\hline $0.2 \%$ Q sm-LCN & $268.7 \pm 0.55$ & $0.419 \pm 0.03$ & $22.3 \pm 0.96$ & $98.5 \pm 0.46$ \\
\hline
\end{tabular}

Each value is presented as mean \pm standard deviation (SD), $\mathrm{N}=3$

Table 2. Inherent energy attributes of monoolein-quercetin complex calculated using static lattice atomistic simulations in vacuum.

\begin{tabular}{|c|c|c|c|c|}
\hline $\begin{array}{l}\text { Energy } \\
\text { component }\end{array}$ & $(\mathrm{MO})_{2}$-Quer & $(\mathrm{MO})_{2}$ & Quer & $\Delta \mathrm{E}^{\mathrm{a}}$ \\
\hline Total $^{b}$ & 3.761 & 6.580 & 13.075 & -15.894 \\
\hline Bond $^{\mathrm{c}}$ & 1.038 & 0.428 & 0.792 & -0.182 \\
\hline Angle $^{d}$ & 3.669 & 3.592 & 1.369 & -1.292 \\
\hline Dihed $^{\mathrm{e}}$ & 4.590 & 3.002 & 3.458 & -1.87 \\
\hline $\mathrm{vdW}^{\mathrm{f}}$ & -4.792 & -0.442 & 8.183 & -12.533 \\
\hline H-bond $\mathrm{g}$ & -0.743 & 0.00 & -0.728 & -0.015 \\
\hline
\end{tabular}

${ }^{a} \Delta \mathrm{E}_{(\mathrm{A} / \mathrm{B})}=\mathrm{E}_{(\mathrm{A} / \mathrm{B})}-\left[\mathrm{E}_{(\mathrm{A})}+\mathrm{E}_{(\mathrm{B})}\right]$; btotal steric energy for an optimized structure; ${ }^{c}$ bond stretching contributions; dbond angle contributions; 'torsional contribution arising from deviations from optimum dihedral angles; fvan der Waals interactions; ghydrogen-bond energy function; values in green represent the structure stabilizing contribution; and values in red represent the structure destabilizing contribution. All values in $\mathrm{kcal} / \mathrm{mol}$.

Table 3. Inherent energy attributes of monoolein-chitosan complex calculated using static lattice atomistic simulations in vacuum.

\begin{tabular}{|l|l|l|l|l|}
\hline $\begin{array}{l}\text { Energy } \\
\text { component }\end{array}$ & $(\mathrm{MO})_{2}$-Cht & $(\mathrm{MO})_{2}$ & Cht & $\Delta \mathrm{E}^{\mathrm{a}}$ \\
\hline Total $^{\mathrm{b}}$ & 2.701 & 6.58 & 6.457 & -10.336 \\
\hline Bond $^{\mathrm{c}}$ & 1.517 & 0.428 & 1.064 & 0.025 \\
\hline
\end{tabular}




\begin{tabular}{|l|l|l|l|l|}
\hline Angle $^{\mathrm{d}}$ & 9.028 & 3.592 & 5.782 & -0.346 \\
\hline Dihed $^{\mathrm{e}}$ & 15.563 & 3.002 & 8.567 & 3.994 \\
\hline vdW $^{\mathrm{f}}$ & -4.388 & -0.442 & 6.189 & -10.135 \\
\hline Elec $^{\mathrm{g}}$ & -19.021 & 0.00 & -15.145 & -3.876 \\
\hline
\end{tabular}

${ }^{\mathrm{a}} \Delta \mathrm{E}_{(\mathrm{A} / \mathrm{B})}=\mathrm{E}_{(\mathrm{A} / \mathrm{B})}-\left[\mathrm{E}_{(\mathrm{A})}+\mathrm{E}_{(\mathrm{B})}\right]$; b total steric energy for an optimized structure; cbond stretching contributions; dbond angle contributions; 'torsional contribution arising from deviations from optimum dihedral angles; ${ }^{f}$ van der Waals interactions; gelectrostatic interactions; values in green represent the structure stabilizing contribution; and values in red represent the structure destabilizing contribution. All values in $\mathrm{kcal} / \mathrm{mol}$. 


\section{Conflict of interests}

The authors declare that there is no conflict of interests. 